\title{
Macrophage-mediated gliadin degradation and concomitant IL-27 production drive IL-10- and IFN- $\gamma$-secreting Tr1-like-cell differentiation in a murine model for gluten tolerance
}

\author{
MA van Leeuwen ${ }^{1,5}$, LMM Costes ${ }^{1,5}$, LA van Berkel ${ }^{1}$, Y Simons-Oosterhuis ${ }^{1}$, MF du Pré ${ }^{1,2}$, AE Kozijn ${ }^{1}$, \\ HC Raatgeep ${ }^{1}$, DJ Lindenbergh-Kortleve ${ }^{1}$, N van Rooijen ${ }^{3}$, F Koning ${ }^{4}$ and JN Samsom ${ }^{1}$
}

Celiac disease is caused by inflammatory T-cell responses against the insoluble dietary protein gliadin. We have shown that, in humanized mice, oral tolerance to deamidated chymotrypsin-digested gliadin (CT-TG2-gliadin) is driven by tolerogenic interferon (IFN)- $\gamma$ - and interleukin (IL)-10-secreting type 1 regulatory T-like cells (Tr1-like cells) generated in the spleen but not in the mesenteric lymph nodes. We aimed to uncover the mechanisms underlying gliadin-specific Tr1like-cell differentiation and hypothesized that proteolytic gliadin degradation by splenic macrophages is a decisive step in this process. In vivo depletion of macrophages caused reduced differentiation of splenic IFN- $\gamma$ - and IL-10-producing Tr1-like cells after CT-TG2-gliadin but not gliadin peptide feed. Splenic macrophages, rather than dendritic cells, constitutively expressed increased mRNA levels of the endopeptidase Cathepsin D; macrophage depletion significantly reduced splenic Cathepsin $D$ expression in vivo and Cathepsin $D$ efficiently degraded recombinant $\gamma$-gliadin in vitro. In response to CT-TG2-gliadin uptake, macrophages enhanced the expression of II27p28, a cytokine that favored differentiation of gliadin-specific Tr1-like cells in vitro, and was previously reported to increase Cathepsin D activity. Conversely, IL-27 neutralization in vivo inhibited splenic IFN- $\gamma$ - and IL-10-secreting Tr1-like-cell differentiation after CT-TG2-gliadin feed. Our data infer that endopeptidase mediated gliadin degradation by macrophages and concomitant IL-27 production drive differentiation of splenic gliadin-specific Tr1-like cells.

\section{INTRODUCTION}

Celiac disease (CD) is driven by a chronic inflammatory T-cell response against the dietary protein gliadin, which results in small intestinal damage in genetically predisposed individuals. $\mathrm{CD}$ is a common food intolerance, affecting $1 \%$ of the Caucasian population. The only effective treatment is a strict gluten-free diet. ${ }^{1}$

Gluten are storage proteins in the grains of wheat, barley and rye. Wheat gluten is a mixture of glutenins and gliadins. Gluten is insoluble in water and resistant to degradation by gastrointestinal enzymes owing to the high proline content, thereby making it possible for large immunogenic peptides to reach the intestinal mucosal surface., ${ }^{2,3}$ In the intestine, gluten is transported across the epithelial barrier to the lamina propria, probably as proteolytic fragments. Transglutaminase 2 (TG2, tissue transglutaminase) facilitates the binding of gluten peptides to the human leukocyte antigen (HLA) class II molecules by the introduction of negative charges in gluten peptides, a process called deamidation. In $\mathrm{CD}$ patients, deamidated gluten peptides are recognized and presented in the context of HLA class II molecules DQ2 or DQ8 to glutenspecific $\mathrm{CD}^{+}{ }^{+} \mathrm{T}$ cells. ${ }^{4,5}$ These gluten-specific $\mathrm{CD} 4^{+} \mathrm{T}$ cells

${ }^{1}$ Laboratory of Pediatrics, Division of Gastroenterology and Nutrition, Erasmus Medical Center-Sophia Children's Hospital, Rotterdam, The Netherlands. ${ }^{2}$ Center for Immune Regulation, Institute of Immunology, University of Oslo and Oslo University Hospital -Rikshospitalet, Oslo, Norway. ${ }^{3}$ Department of Molecular Cell Biology and Immunology, VU University Medical Center, Amsterdam, The Netherlands and ${ }^{4}$ Department of Immunohematology and Blood Transfusion, Leiden University Medical Center, Leiden, The Netherlands. Correspondence: JN Samsom (j.samsom@erasmusmc.nl)

${ }^{5}$ These authors share first authorship. 
are inflammatory, produce interferon (IFN) $-\gamma$ and interleukin (IL)-21 and drive mucosal damage. ${ }^{6-9}$ Crucially, inflammatory gluten-specific $\mathrm{CD} 4{ }^{+} \mathrm{T}$ cells can be detected in $\mathrm{CD}$ patients but not in healthy HLA-DQ2 ${ }^{+}$individuals who tolerate gluten. ${ }^{8}$ Despite the increasing knowledge on CD pathogenesis, it is still unknown why oral tolerance to gluten is lost in HLA-DQ2 ${ }^{+}$ $\mathrm{CD}$ patients while the majority of HLA-DQ2 ${ }^{+}$individuals remain tolerant to gluten.

To identify the processes that underlie oral tolerance to gluten, we have made use of double transgenic mice that express HLA-DQ2 and an HLA-DQ2-restricted T-cell receptor (DQ2.gliadinTCR) derived from a $\mathrm{T}$ cell of a $\mathrm{CD}$ patient and that is specific for the DQ2.5-glia- $\gamma 1$-epitope. ${ }^{10}$ In these DQ2.gliadinTCR mice, feeding deamidated gliadin induces no sign of CD pathology, but instead gliadin-specific tolerance is mounted in the spleen where suppressive IL-10-secreting type 1 regulatory T (Treg)-like cells (Tr1-like cells) are formed. ${ }^{10}$ This was unexpected as, in general, oral tolerance to harmless soluble food proteins, such as ovalbumin (OVA), is dependent on antigen presentation in the intestinal mucosa-draining lymphoid tissue, an environment specifically adapted to favor differentiation of regulatory responses. In particular, after OVA feed, the antigen is transported to mesenteric lymph nodes (MLNs) and Peyer's patches, presented by specialized mucosal antigen-presenting cells (APCs), which induce the differentiation of Foxp3 ${ }^{+}$Treg cells that drive protein-specific tolerance. ${ }^{11-14}$ In contrast, the tolerogenic T-cell response to deamidated gliadin in DQ2.gliadinTCR mice does not occur under the strict control of mucosa-resident dendritic cells (DCs) and the mucosal lymph node microenvironment but predominantly occurs in the spleen. Differentiating gliadinspecific $T$ cells do not upregulate Foxp3 expression but produce IFN- $\gamma$ and IL-10. ${ }^{10}$ These data suggest that oral tolerance to gliadin may be mechanistically different from tolerance to some other known food proteins and may depend more on suppression by IL-10-secreting Tr1-like cells.

The aim of this study was to identify the mechanisms by which orally administered gliadin elicits gliadin-specific T-cell proliferation and differentiation of Tr1-like cells in the spleen. In particular, as macrophages contain higher levels of lysosomal proteases than DCs and rapidly degrade internalized proteins, ${ }^{15}$ we compared the role of macrophages and DCs in this process. We report that macrophages rather than DCs have a pivotal role in the induction of gliadin-specific Tr1-like cells in the spleen possibly through their ability to express endopeptidases, among which is Cathepsin D, that can cleave gliadin. Concomitantly, gliadin degradation by macrophages elicits Il27p28 expression. IL-27 is a cytokine reported to enhance Cathepsin D activity and is required for enhancing Tr1-cell differentiation in vivo. ${ }^{16,17}$

\section{RESULTS}

Ingested gliadin is detected in $\mathrm{CD}_{11 \mathrm{C}^{+}}$cells in the MLNs but does not induce strong T-cell proliferation

Previously, using the DQ2.gliadinTCRtg T-cell transfer model we have shown that orally administered chymotrypsin-digested transglutaminase 2-treated (CT-TG2) fragmented gliadin induced a dominant Tr1-like-cell differentiation in the spleen but did not elicit substantial proliferation in the MLNs. ${ }^{10} \mathrm{We}$ hypothesized that the absence of T-cell proliferation in the MLNs was related to a reduced ability of APCs to present fragmented CT-TG2-gliadin owing to its resistance to proteolysis. This hypothesis would predict that the protein is detectable in the MLNs of DQ2.gliadinTCR mice after oral administration. To investigate whether CT-TG2-gliadin is present in the MLNs after feed, HLA-DQ2.MHCII ${ }^{\Delta / \Delta}$ (DQ2) mice received CT-TG2-gliadin per gavage or intravenously (i.v.) as a positive control. After $18 \mathrm{~h}$, lymphoid organs and spleens were analyzed by microscopy for the presence of gliadin by immunofluorescence using an anti-gliadin antibody (Ab). After gavage, gliadin-positive cells were detectable in the MLNs and spleen while no signal was detected in mice that did not receive gliadin gavage (Figure 1a and see Supplementary Figure S1 online).

To further substantiate the presence of gliadin-positive cells in the MLNs, mice were gavaged with fluorescently labeled CT-TG2-gliadin or non-labeled CT-TG2-gliadin as a negative control or i.v. injected with fluorescently labeled CT-TG2-gliadin as a positive control. At $18 \mathrm{~h}$ after gavage or i.v. injection, MLNs were analyzed by flow cytometry. As shown in Figure $\mathbf{1 b}$, after gavage Alexa Fluor 647-labeled CT-TG2-gliadin was associated with $\mathrm{CD} 11 \mathrm{c}^{+}$cells in the MLNs. In DQ2 mice, the CD11 $\mathrm{c}^{+}$Alexa Fluor $647^{+}$cells co-expressed CD103 but not CD11b or CD8 $\alpha$ (Figure 1c). B220-positive B cells were negative for Alexa Fluor 647-labeled CT-TG2-gliadin in the MLNs (Figure 1d). Of note, however, in wild-type C57BL/6 mice, gavaged Alexa Fluor

\footnotetext{
Figure 1 Ingested CT-TG2-gliadin is detectable in the mesenteric lymph nodes (MLNs). (a) DQ2 mice received 3.2 mg CT-TG2-gliadin by intragastric gavage (i.g.) (left and middle panels) or no gavage for negative control staining (right panel). At $18 \mathrm{~h}$ after gavage, MLNs (left) and spleen (middle) were isolated and cryosections were stained with an anti-gliadin primary antibody, Dylight-488 labeled conjugate and counterstained with 4,6-diamidino-2phenylindole (DAPI). Representative immunofluorescence stainings of MLNs and spleen are shown. Green indicates the anti-gliadin staining and blue indicates the nuclear counterstaining with DAPI. Original magnification: $\times 20 . n=2$ mice. See Supplementary Figure S1 for additional controls. (b-d) Mice received $3.2 \mathrm{mg}$ CT-TG2-gliadin i.g., either unlabeled or labeled with Alexa Fluor 647 succinimidyl ester or received $1.0 \mathrm{mg}$ Alexa Fluor 647-labeled CT-TG2-gliadin intravenously. (b, c) At $18 \mathrm{~h}$ after gavage, single-cell suspensions of MLNs were depleted of B and CD4 ${ }^{+}$T cells and stained for $7-$ aminoactinomycin D (7AAD), CD45, CD11c, CD11b, CD103, and CD8 $\alpha$ and analyzed for Alexa Fluor 647. (b) Representative dot plots of stainings gated on $7 A A D^{\text {neg }} C D 45^{+}$, showing the positivity for $\mathrm{CD} 11 \mathrm{c}$ and Alexa Fluor 647. (c) Representative dot plots showing the positivity for Alexa Fluor 647 in $7 \mathrm{AAD}{ }^{\text {neg }} \mathrm{CD} 45^{+} \mathrm{CD} 11 \mathrm{c}^{+}$cells subsequently gated for CD103, CD11b, and CD8 $\alpha$. Gates were set using the unlabeled CT-TG2-gliadin controls. $N=3$ per group. (d) At $18 \mathrm{~h}$ after gavage, a single-cell suspension of MLNs was stained for B220 and analyzed for Alexa Fluor 647 . Representative dot plots of stainings showing $7 \mathrm{AAD}{ }^{\text {neg }} \mathrm{B} 220^{+} \mathrm{DQ} 2^{+}$cells.
} 
647-labeled CT-gliadin was preferentially associated with $\mathrm{CD}_{103}{ }^{+} \mathrm{CD}_{11 \mathrm{~b}^{-}}$that were $\mathrm{CD} 8 \alpha^{+}$(see Supplementary Figure 1b). The fact that the labeled gliadin does not always co-localize with $\mathrm{CD} 8 \alpha^{+}$DCs may be related to the variable frequency of $\mathrm{CD}_{103}{ }^{+} \mathrm{CD} 11 \mathrm{~b}^{-} \mathrm{CD} 8 \alpha^{+}$DCs observed in DQ2 mice (data not shown). a

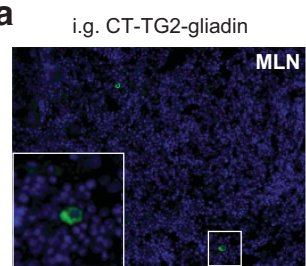

b

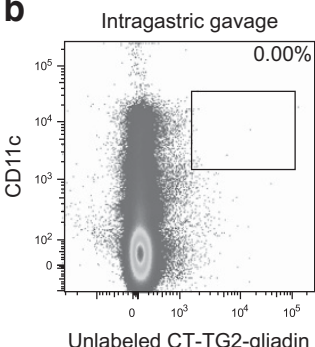

C
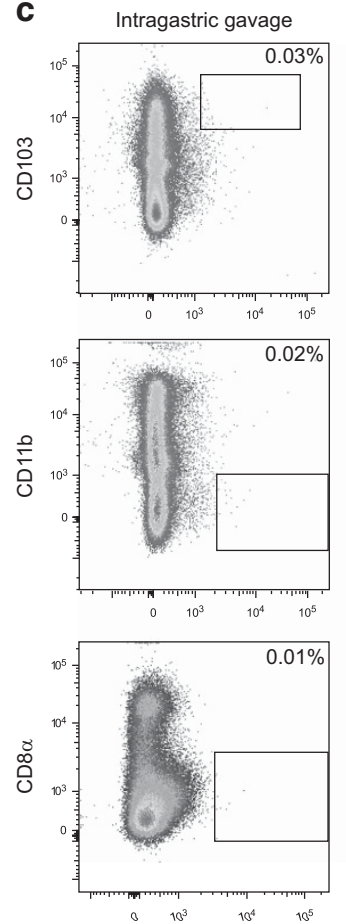

Unlabeled CT-TG2-gliadin

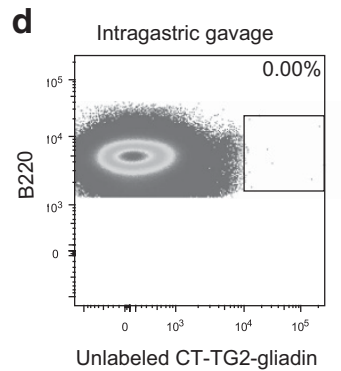

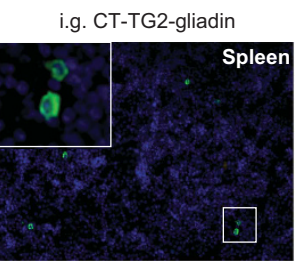
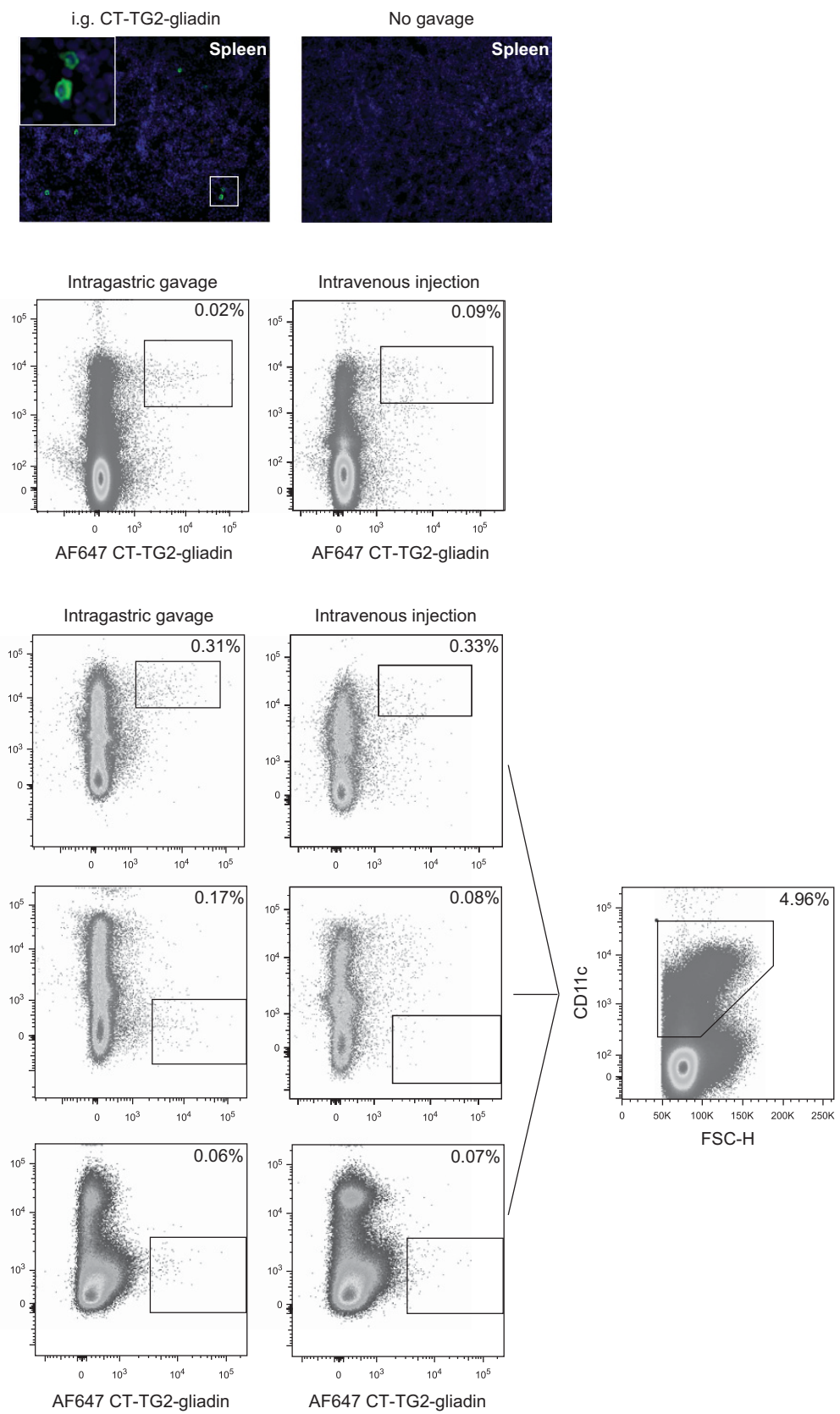

AF647 CT-TG2-gliadin

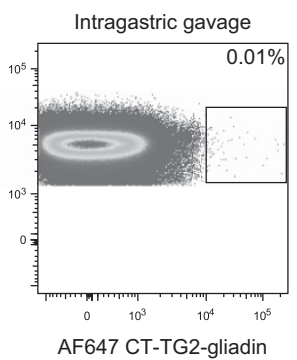


In conclusion, after oral CT-TG2-gliadin administration the protein is present in the MLNs and associated with $\mathrm{CD} 11 \mathrm{c}^{+}$ $\mathrm{CD}_{103}{ }^{+} \mathrm{CD}_{11 \mathrm{~b}}{ }^{-}$cells.

\section{Ingested TG2-gliadin peptide is presented efficiently in the MLNs}

As, in contrast to the soluble protein OVA, CT-TG2-gliadin gavage induces preferential T-cell proliferation in the spleen rather than the MLNs, we hypothesized that proteolysis of gliadin into smaller peptides and loading into the DQ2 molecules may be insufficient to induce a detectable T-cell proliferation in the MLNs. Consequently, the gliadin-specific T-cell response was investigated in the MLNs by bypassing endosomal degradation and major histocompatibility complex II (MHCII) loading using gavage of synthetic TG2-gliadin peptides in the DQ2.gliadinTCRtg T-cell transfer model as previously described. ${ }^{10}$ In short, $\mathrm{CD} 4{ }^{+}$gliadin-TCRtg T cells were purified from DQ2.gliadinTCR mice, labeled with 5,6carboxy-succinimidyl-fluoresceine-ester (CFSE) and i.v. injected in DQ2 acceptor mice. Starting the day after T-cell transfer, DQ2 mice raised on a gluten-free diet received either $3 \mathrm{mg}$ TG2-gliadin peptide per gavage on 3 consecutive days or $60 \mathrm{mg}$ CT-TG2-gliadin distributed over 3 gavages on the first day. At $72 \mathrm{~h}$ after the first gavage, MLNs and spleen were isolated and examined for the division of transferred gliadinspecific T cells. Confirming earlier data, ${ }^{10}$ CT-TG2-gliadin feed preferentially induced $\mathrm{T}$-cell proliferation in the spleen when compared with the MLNs (Figure 2a). However, in contrast to CT-TG2-gliadin, TG2-gliadin peptide also induced T-cell proliferation in the gut-draining MLNs demonstrating that smaller fragments of gliadin can be directly presented by DQ2positive MLN APCs to induce substantial T-cell proliferation in the MLNs (Figure 2a). The number of divisions and the percentage of $\mathrm{V} \beta 1^{+}$cells per division were comparable between MLNs and spleen after TG2-gliadin peptide feed (Figure 2b).

Within the mucosal environment of the MLNs, presentation of the harmless food protein OVA by $\mathrm{CD}_{103}{ }^{+}$DCs preferentially induces differentiation of naive $\mathrm{T}$ cells into Foxp $3^{+}$Treg cells. ${ }^{11-14}$ Therefore, we anticipated that the TG2-gliadin peptide-induced T-cell response in the MLNs may have an enhanced frequency of Foxp $3^{+}$T cells. However, TG2gliadin peptide did not induce preferential Foxp $3^{+}$T-cell differentiation in the MLNs as the percentage of gliadin-specific Foxp $3^{+} \mathrm{T}$ cells in the MLNs was not higher than in the spleen (Figure 2c). There could be multiple possible explanations for the absence of preferential Foxp $^{+}$cell differentiation.
Differentiation of peripheral Tregs is determined by intrinsic characteristics of the naive $\mathrm{T}$ cell, including peptide MHC ligand affinity, as well as extrinsic factors, including the nature of the APC and location of the antigen presentation. It appears that our finding was not due to a total inability of the transgenic MLN-DC to induce Foxp $3^{+} \mathrm{T}$ cells, as gliadin-specific Foxp $3^{+} \mathrm{T}$ cells differentiated in vitro in response to antigen presentation by MLN-DC cultured with high concentrations of transforming growth factor- $\beta$ and retinoic acid (Figure 2d).

In conclusion, in contrast to fragmented CT-TG2-gliadin, cognate TG2-gliadin peptide feed induces efficient T-cell proliferation in the MLNs, suggesting that insufficient endosomal degradation and/or MHC loading of gliadin in the MLN APCs may account for the absence of T-cell proliferation in response to CT-TG2-gliadin.

\section{Macrophages determine the differentiation of splenic gliadin-specific Tr1-like cells in vivo}

Macrophages, in particular marginal zone macrophages in the spleen, have a role in capturing and processing antigen. ${ }^{18}$ When compared with DCs, these macrophages have higher levels of lysosomal proteases and degrading capacity. ${ }^{15}$ Therefore, we examined the contribution of macrophages to splenic gliadin presentation by depletion of macrophages with clodronate liposomes in the DQ2.gliadinTCRtg T-cell transfer model. Previously, it was shown that infusion of clodronate liposomes effectively depletes macrophages by accumulation of clodronate in the cell leading to apoptosis. ${ }^{19}$ DQ2 mice were i.v. injected with clodronate liposomes 1 day prior to $\mathrm{CD} 44^{+}$ DQ2.gliadinTCRtg T-cell transfer. I.v. injection of saline was used as a negative control. As observed previously, ${ }^{20}$ histological evaluation at 3 days after clodronate liposome treatment revealed that $\mathrm{CD} 169^{+}$cells were efficiently depleted from the spleen (Figure 3a). CD11 $c^{+}$cells were nevertheless detectable in the spleen of liposome-injected mice (Figure 3b). After injection of clodronate liposomes or saline and subsequent transfer of $\mathrm{CD}^{+}{ }^{+}$gliadin-TCRtg T cells, the mice were fed CT-TG2-gliadin or TG2-gliadin peptide. At $72 \mathrm{~h}$ after feed, the spleens were isolated and division of transferred gliadinspecific $\mathrm{T}$ cells was examined. Flow cytometric analysis revealed that clodronate treatment did not inhibit proliferation of $\mathrm{CD}^{+}$gliadin-TCRtg $\mathrm{T}$ cells in the spleen of mice after CT-TG2-gliadin feed (Figure 3c,d). Indeed, equal percentages of transferred cells had divided into both groups and the cells had undergone the same number of divisions. As expected, proliferation in response to TG2-gliadin peptide feed was also

Figure 2 Ingested gliadin peptide is presented efficiently in the mesenteric lymph nodes (MLNs) and spleen. (a, b) DQ2 mice received $1 \times 10^{7}$ carboxyfluorescein succinimidyl ester (CFSE)-labeled CD4 ${ }^{+}$gliadin-TCRtg T cells and, 1 day after transfer, received 60 mg CT-TG2-gliadin distributed over three intragastric gavages (i.g.) on the same day or received i.g. TG2-gliadin peptide 3 mg day ${ }^{-1}$ on three consecutive days. At 72 h, MLNs and spleens were isolated and analyzed by flow cytometry for division of transferred cells. For each group, a representative histogram plot showing CFSE dilution gated on CD4 ${ }^{+} \mathrm{V} \beta 1^{+}$cells is depicted. For TG2-gliadin peptide gavaged mice, the percentage of $\mathrm{CD} 4{ }^{+} \mathrm{V} \beta 1^{+}$cells in each peak of division is shown as mean \pm s.d. for five mice. (c) Representative dot plots of Foxp3 expression on dividing CD4 ${ }^{+} \mathrm{V} \beta 1^{+}$cells from the MLNs and spleen at $72 \mathrm{~h}$ after the first TG2-gliadin peptide feed. Representative for $n=5$ mice. (d) Splenocytes from DQ2.gliadinTCR mice were cultured with 0.5 mg ml ${ }^{-1}$ CT-TG2-gliadin in the presence or absence of $20 \mathrm{ng} \mathrm{ml}^{-1}$ recombinant human transforming growth factor- $\beta$ (TGF- $\beta$ ) with or without $10 \mathrm{~nm}$ retinoic acid for $72 \mathrm{~h}$. Proliferation was assessed by CFSE dilution, Foxp3 expression was determined by fluorescence-activated cell sorting analysis. 
a

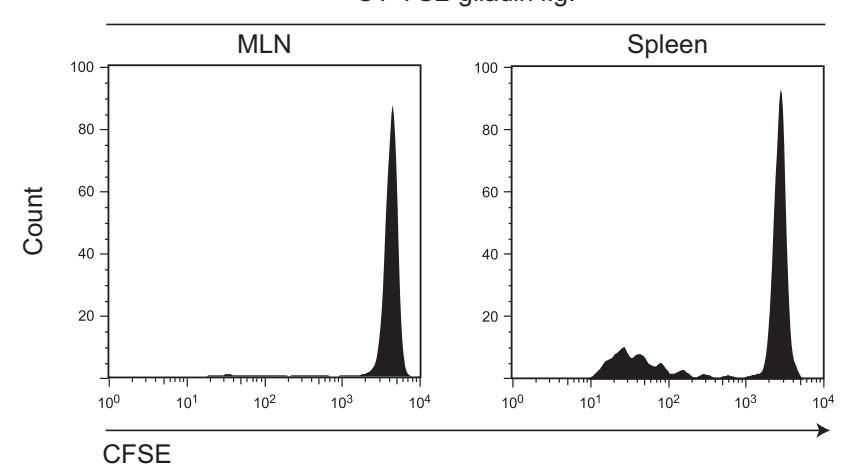

TG2-gliadin peptide i.g.

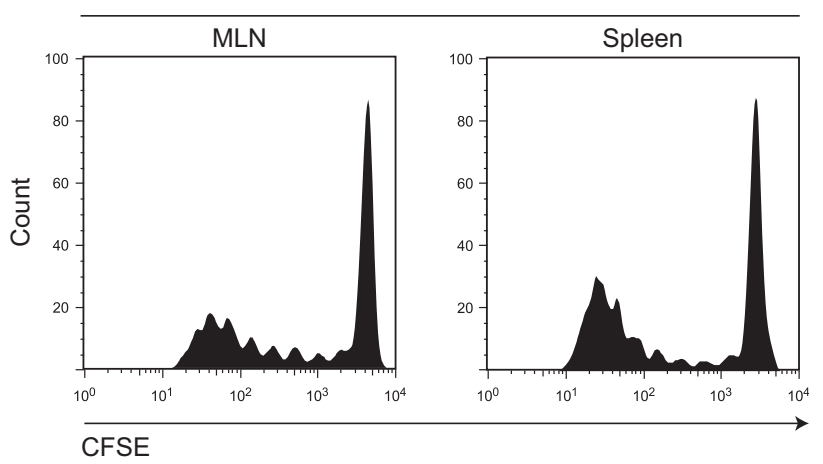

b TG2-gliadin peptide i.g.

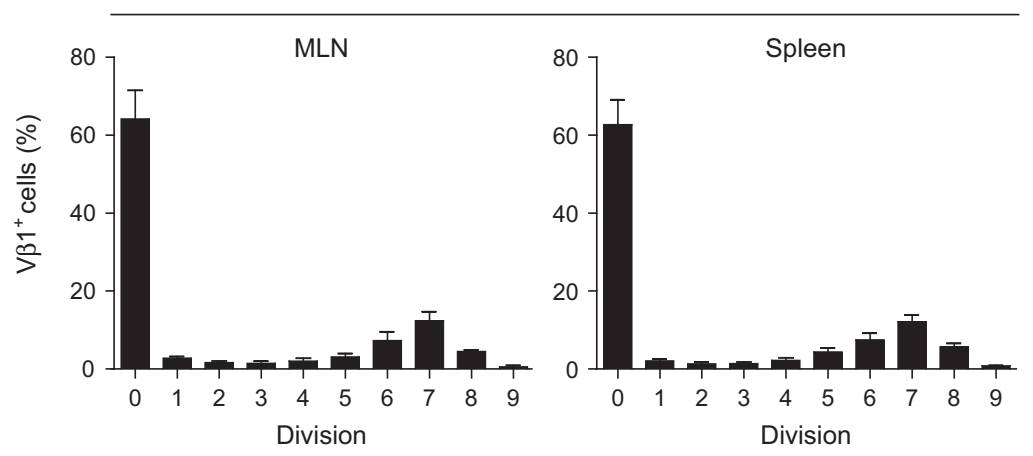

C
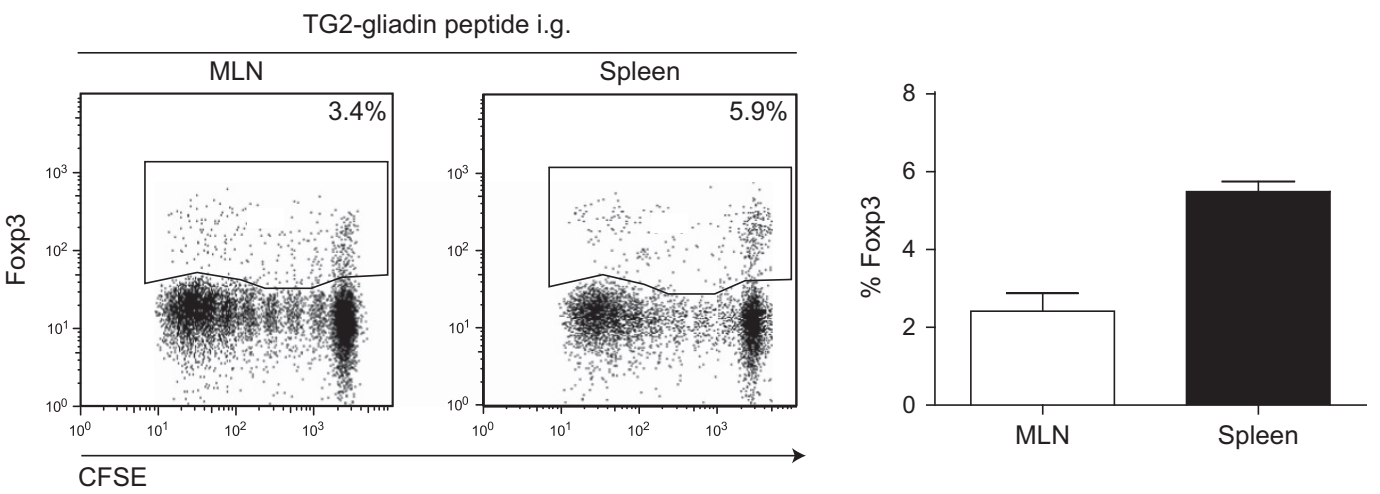

d

CT-TG2-gliadin

CT-TG2-gliadin + TGF- $\beta$

CT-TG2-gliadin
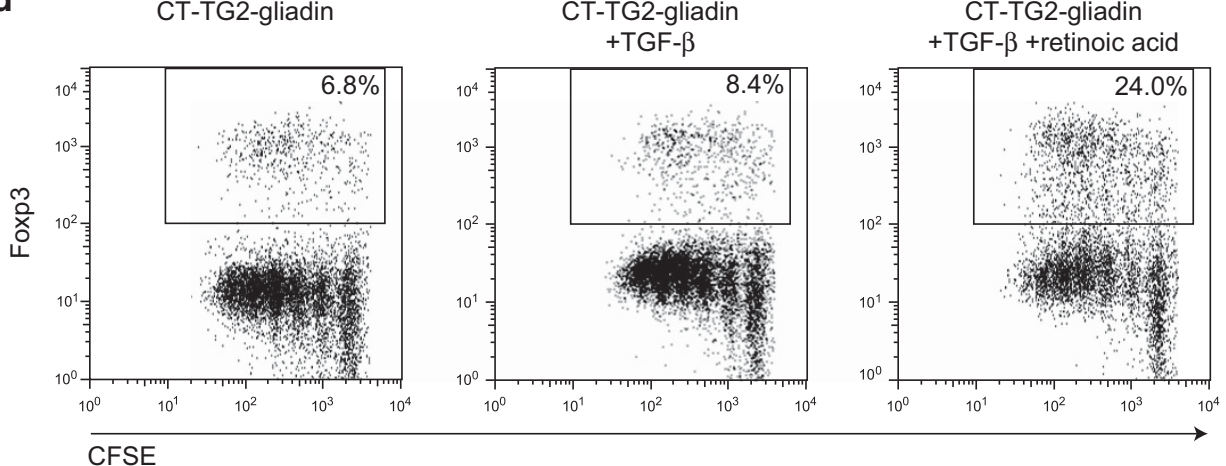
a

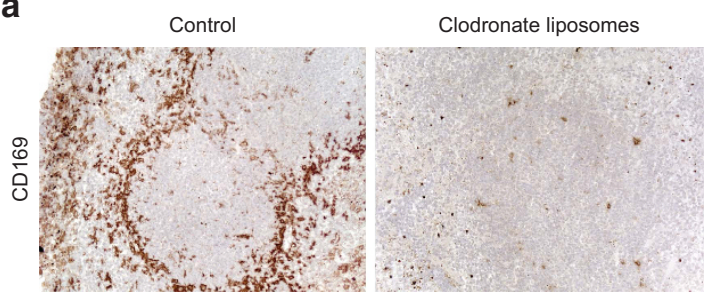

b
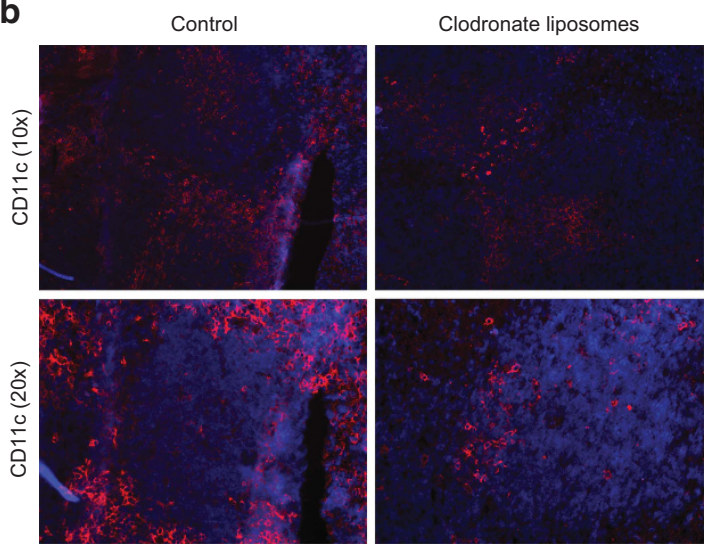

e

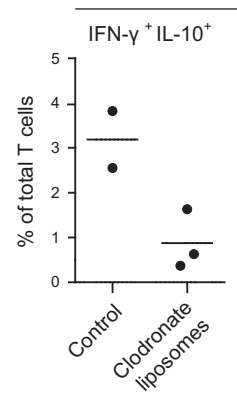

CT-TG2-gliadin
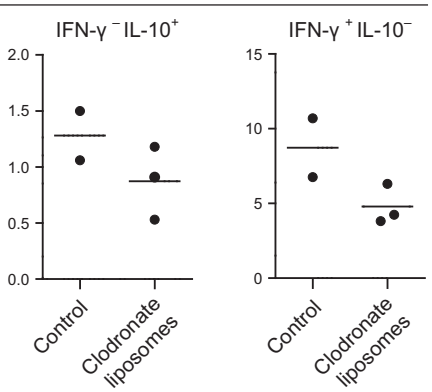

g

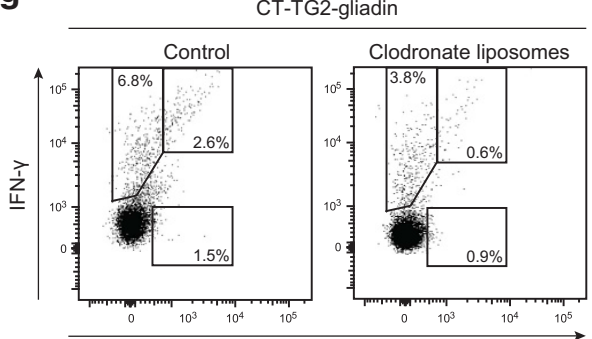

IL-10

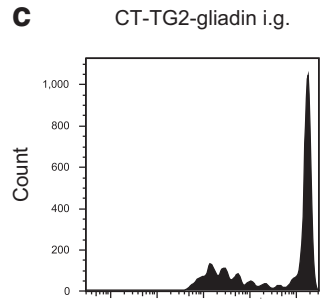

TG2-gliadin peptide i.g.
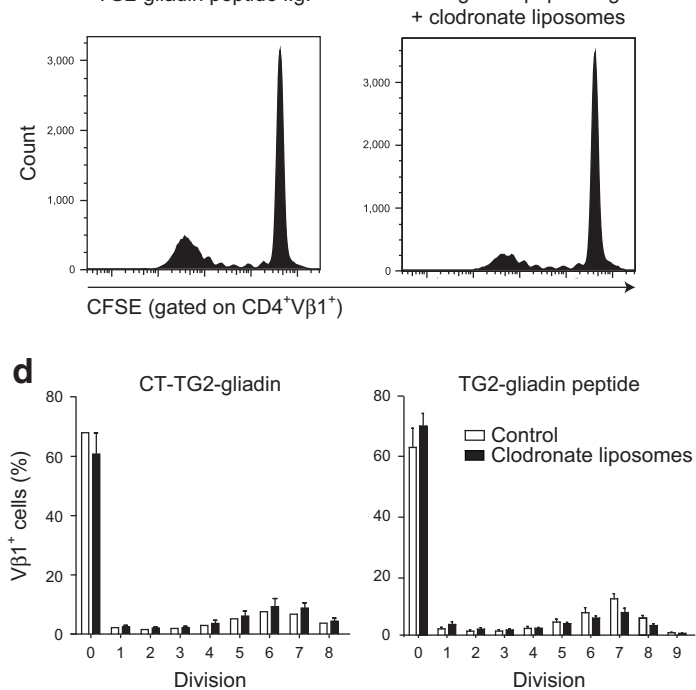

f

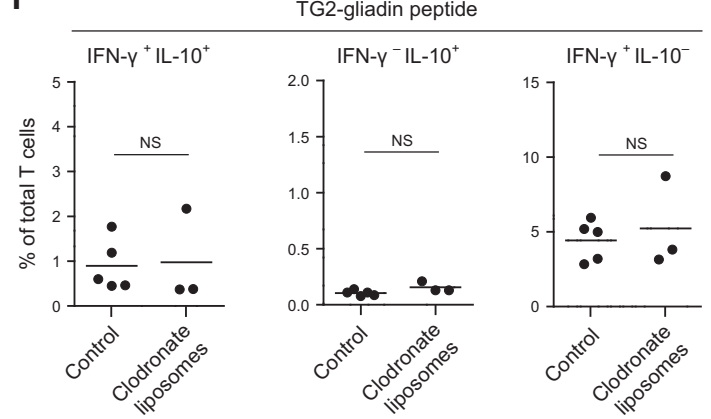

h

TG2-gliadin peptide

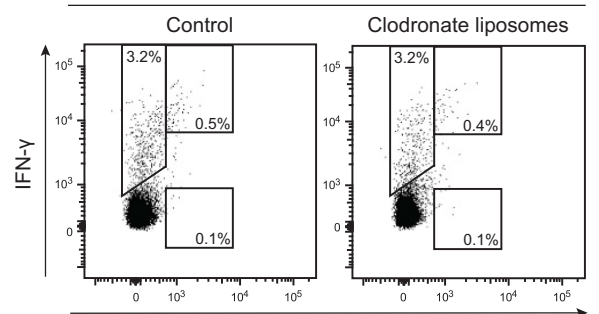

IL-10

Figure 3 Macrophages determine the differentiation of splenic gliadin-specific Tr1-like cells in vivo. (a-h) DQ2 mice received $1 \times 10^{7}$ carboxyfluorescein succinimidyl ester (CFSE)-labeled CD4 ${ }^{+}$gliadin-TCRtg T cells and, 1 day after transfer, received 75 mg CT-TG2-gliadin distributed over three intragastric gavages (i.g.) on the same day or received i.g. TG2-gliadin peptide $3 \mathrm{mg}$ and $6 \mathrm{mg}$ during two consecutive days. At $24 \mathrm{~h}$ prior to the T-cell transfer, mice were either injected with $200 \mu \mathrm{l}$ clodronate liposomes or saline (control). At $72 \mathrm{~h}$ after the first gavage, spleens were isolated and single-cell suspensions were analyzed by flow cytometry for division of transferred cells. (a) Representative CD169 stainings on spleen sections of control (left) and clodronate liposome (right) injected mice. $N=7$ control mice, $n=6$ clodronate liposome mice. (b) Representative CD11c staining on spleen sections of control (left) and clodronate liposome (right) injected mice. (c) Representative histograms showing CFSE dilution on CD4 ${ }^{+} \mathrm{V} \beta 1^{+}$cells for each group. (d) Bar graphs of dividing $C D 4{ }^{+} \mathrm{V} \beta 1^{+}$cells per division peak. $(n=2-5$ per group) (e, $\mathbf{f})$ Quantitative analysis of the percentage of interferon (IFN)- $\gamma$ - and interleukin (IL)-10-producing cells within restimulated total CD4 ${ }^{+} \mathrm{V} \beta 1^{+}$cells after CT-TG2-gliadin gavage (e) or TG2-gliadin peptide gavage (f). $(\mathbf{g}, \mathbf{h})$ Representative dot plots of intracellular IFN- $\gamma$ and IL- 10 within the total restimulated CD $4^{+} \mathrm{V} \beta 1^{+}$cell population after CT-TG2-gliadin feed (g) or TG2-gliadin peptide feed (h). Each symbol represents one mouse. Horizontal bars represent mean values. Student's $t$-test. NS, not significant. 
unchanged after macrophage depletion (Figure $3 \mathbf{c}, \mathbf{d}$ ). At $72 \mathrm{~h}$, both dividing and non-dividing $\mathrm{CD} 4^{+}$gliadin-TCRtg $\mathrm{T}$ cells were isolated from the spleen and restimulated in vitro with TG2-gliadin peptide. At $24 \mathrm{~h}$ after in vitro restimulation, the percentage of cytokine-secreting gliadin-specific $\mathrm{T}$ cells was analyzed by flow cytometry. Depletion of macrophages before CT-TG2-gliadin feed elicited an approximately threefold reduction in the percentage of IL-10- and IFN- $\gamma$-producing gliadin-specific $\mathrm{T}$ cells in the spleen (Figure $\mathbf{3 e}, \mathbf{g}$ ). In contrast, macrophage depletion before TG2-gliadin peptide feed did not affect the percentage of IL-10- and IFN- $\gamma$-secreting T cells (Figure 3f,h). Further investigation of the proliferating $\mathrm{T}$ cells in the macrophage-depleted CT-TG2-gliadin-fed mice suggested that these cells had a Th0-like phenotype with twofold lower levels of IL-2 secretion and similar low levels of IL-17 and IL-4 when compared with macrophage-sufficient CT-TG2-gliadin-fed mice (data not shown).

In conclusion, in mice fed with CT-TG2-gliadin, splenic macrophages may drive the differentiation of IL-10- and IFN$\gamma$-producing splenic gliadin-specific T cells. Macrophages are not essential for these gliadin-specific $\mathrm{T}$-cell responses when mice are fed TG2-gliadin peptide raising the possibility that macrophage-driven degradation of CT-TG2-gliadin regulates the differentiation of gliadin-specific Tr1-like cells in the spleen.

\section{Macrophages favor the differentiation of gliadin-specific Tr1-like cells in vitro when compared with DCs}

To demonstrate that macrophages are required for directing the phenotype of the proliferating gliadin-specific T cells, we developed an in vitro model for Tr1-like-cell differentiation. In short, naive $\mathrm{CD}^{+}{ }^{+}$gliadin-TCRtg $\mathrm{T}$ cells from DQ2.gliadinTCR mice were co-cultured with freshly isolated splenic macrophages $\left(\mathrm{CD} 11 \mathrm{c}^{\text {neg }} \mathrm{CD} 11 \mathrm{~b}^{+}\right)$or splenic DCs $\left(\mathrm{CD} 11 \mathrm{c}^{+}\right)$ from DQ2 mice in the presence of CT-TG2-gliadin. The ability of splenic macrophages and splenic DCs to induce Tr1-like-cell differentiation in response to CT-TG2-gliadin was assessed by flow cytometry at $72 \mathrm{~h}$ of culture. As it is possible that macrophages and DCs cooperate in inducing the Tr1 cell phenotype, cultures with macrophages only, DCs only, and mixtures of macrophages and DCs were used. After $72 \mathrm{~h}$, DCs cultured in the presence of CT-TG2-gliadin induced a higher frequency of dividing $\mathrm{T}$ cells with a mean of approximately $80 \%$ while macrophages with CT-TG2-gliadin induced approximately $70 \%$ of $\mathrm{T}$ cells to divide. Once dividing, the $\mathrm{T}$ cells a

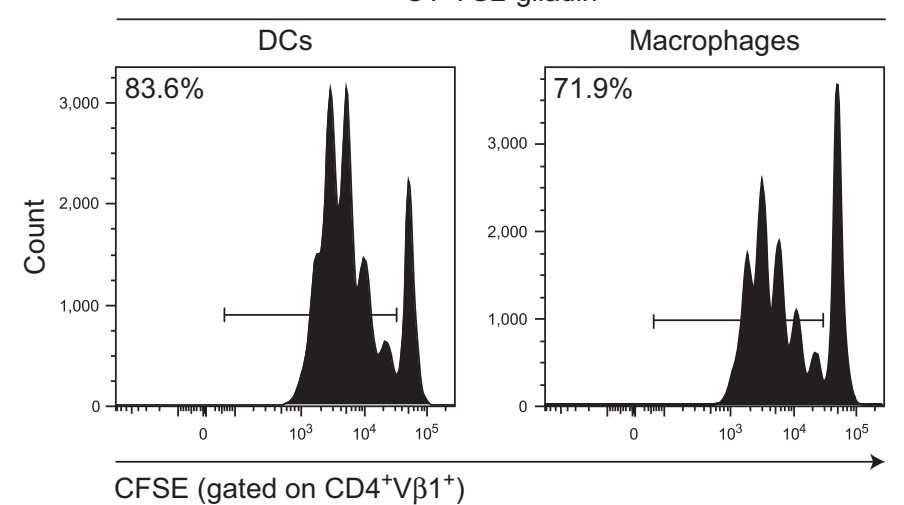

CT-TG2-gliadin

b

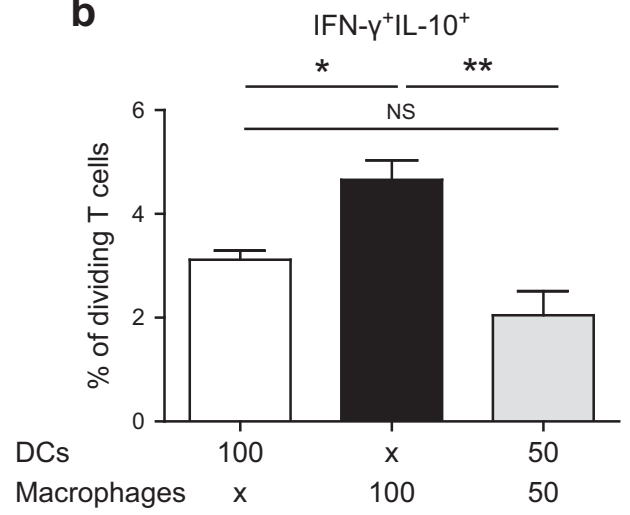

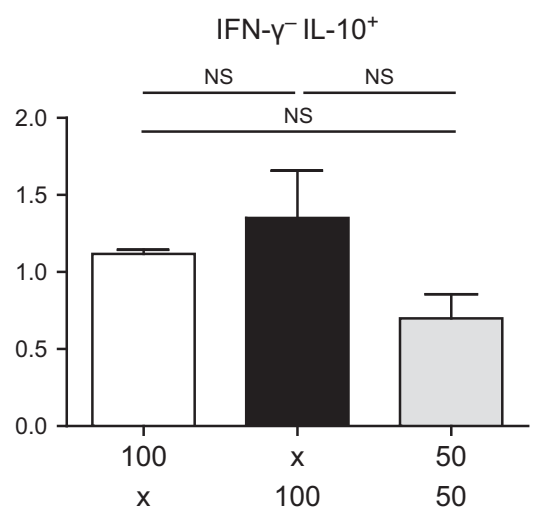

IFN-Y IL-10 $10^{+}$

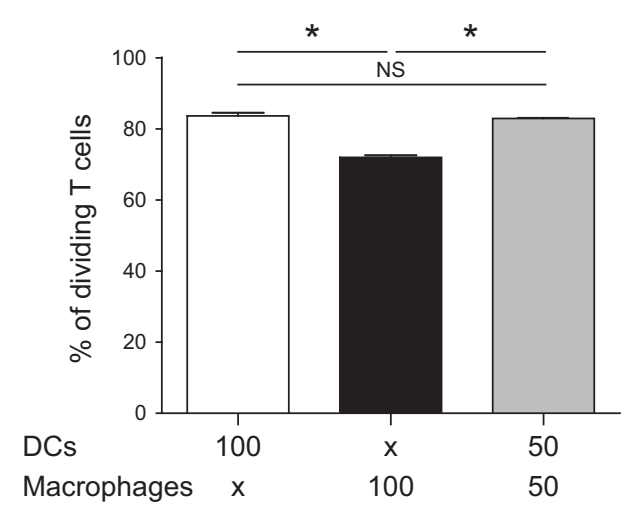

Macrophages $\mathrm{x}$

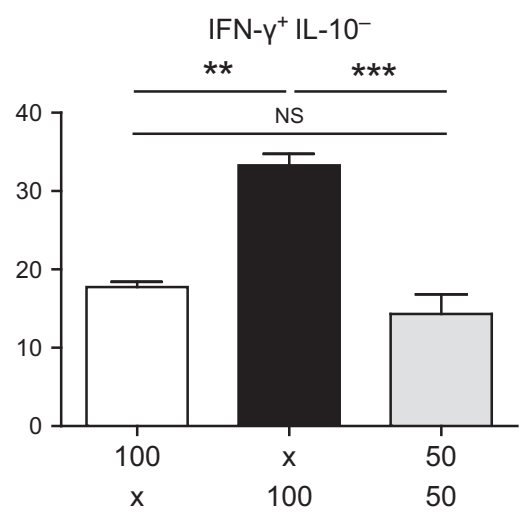

Figure 4 Macrophages determine the differentiation of splenic gliadin-specific Tr1-like cells in vitro. (a, b) CD4 ${ }^{+}$gliadin-TCRtg T cells were purified from the spleens, labeled with carboxyfluorescein succinimidyl ester (CFSE), and stimulated in vitro with CT-TG2-gliadin-loaded splenic macrophages or dendritic cells (DCs) or both. At $72 \mathrm{~h}$, T-cell proliferation and T-cell cytokine production were assessed by flow cytometry. (a) Representative histograms and bar graph showing T-cell proliferation, gated on CD4 ${ }^{+} \mathrm{V} \beta 1^{+} \mathrm{T}$ cells. (b) Quantitative analysis of the percentage of interferon (IFN)- $\gamma$ - and interleukin (IL)-10-producing cells within the dividing $\mathrm{CD} 4^{+} \mathrm{V} \beta 1^{+}$cell population. Horizontal bars represent mean values $\pm \mathrm{s}$.d. ( $n=3$ per group). One-way analysis of variance followed by Bonferroni post-hoc test. NS, not significant. 
a Macrophages
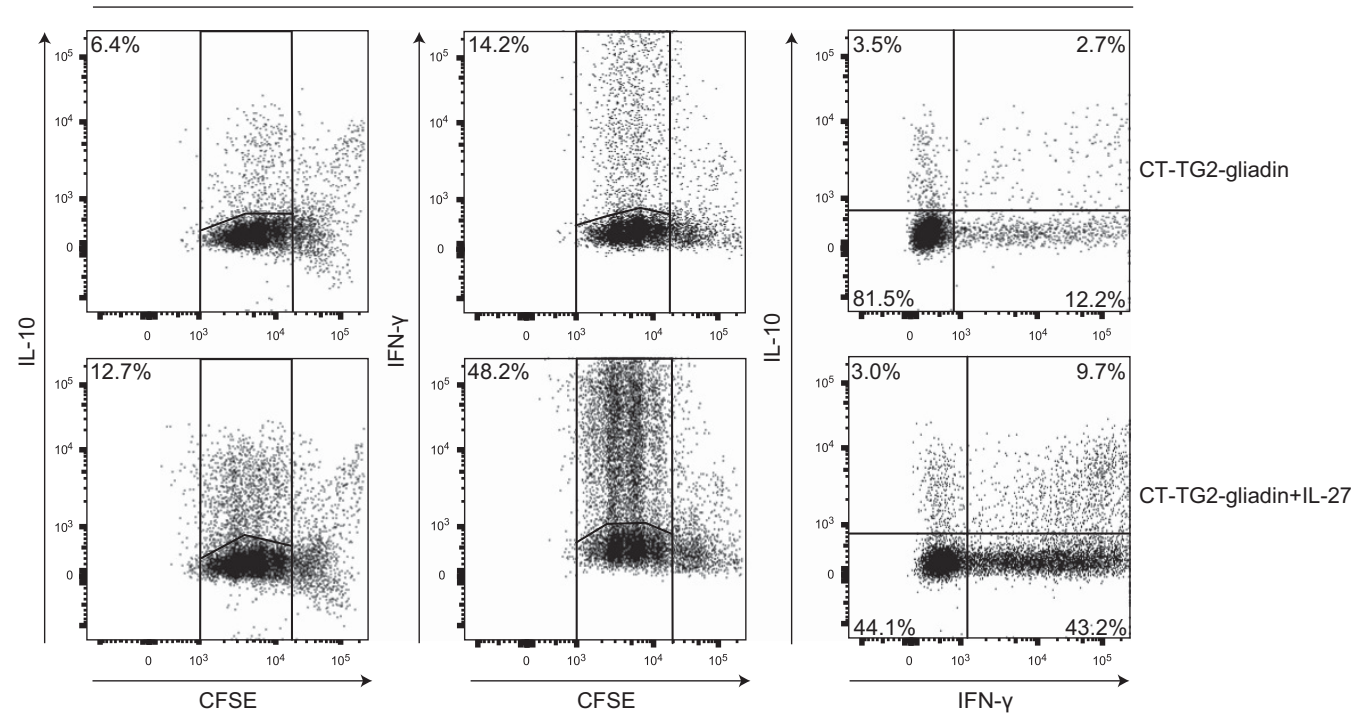

b
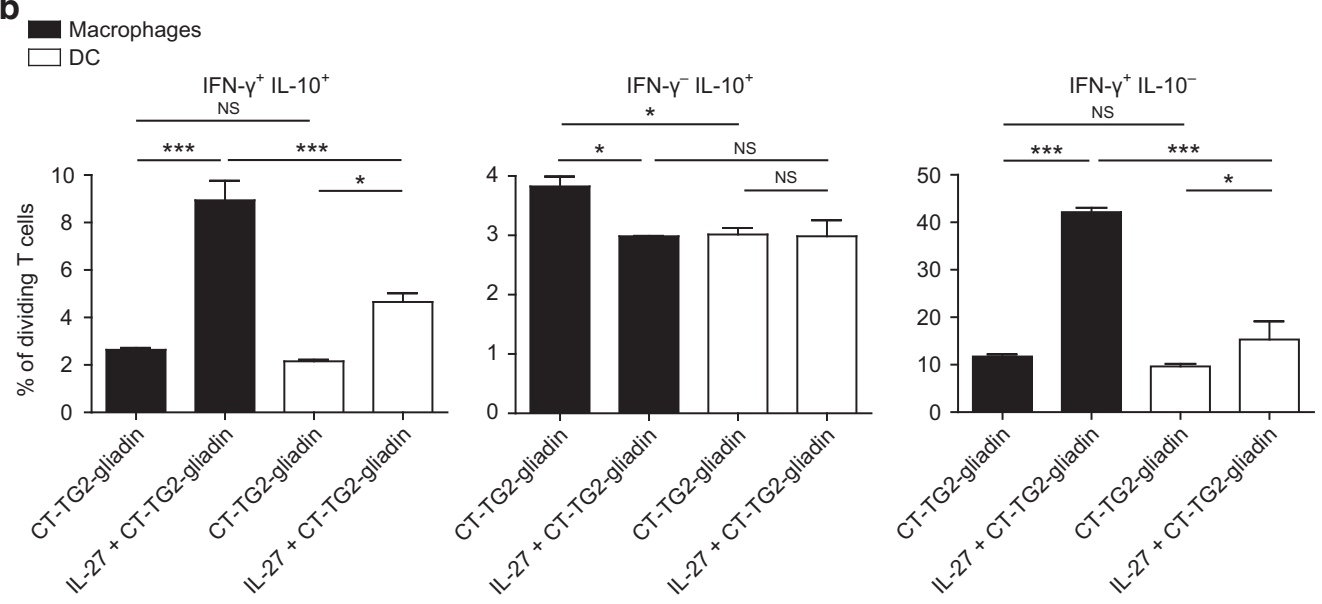

c

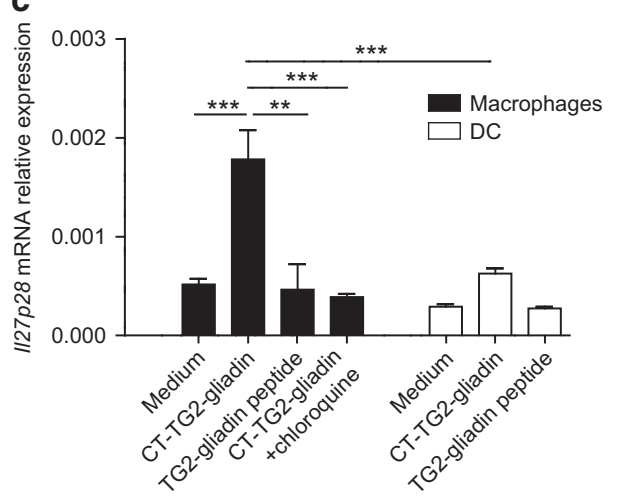

d

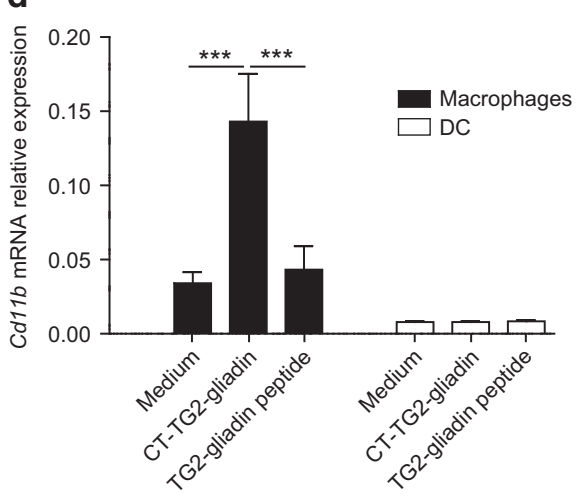

Figure 5 Interleukin (IL)-27 induces IL-10 and interferon (IFN)- $\gamma$ production of gliadin-specific T cells in vitro and is specifically expressed by macrophages in response to gliadin. $(\mathbf{a}, \mathbf{b}) \mathrm{CD} 4^{+}$gliadin-TCRtg T cells were purified from the spleens, labeled with carboxyfluorescein succinimidyl ester

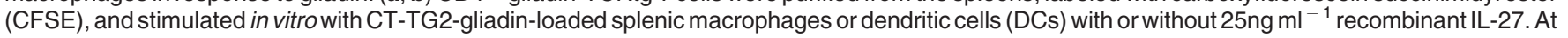
$72 \mathrm{~h}, \mathrm{~T}$-cell proliferation and T-cell cytokine production were assessed by flow cytometric analysis. (a) Representative dot plots showing proliferating IFN- $\gamma$ - and IL-10-producing CD4 ${ }^{+}$T cells after co-culture with splenic macrophages. An isotype control stain was used to set gates. (b) Quantitative analysis of the percentage of cytokine-producing cells within the dividing CD4 ${ }^{+}$cell population. Horizontal bars represent mean values $\pm \mathrm{s}$.d. $(n=3$ per group). One experiment representative of two independent experiments. (c, d) Splenic macrophages or DCs from DQ2 mice were cultured for $24 \mathrm{~h}$ in the presence of CT-TG2-gliadin $\left(0.5 \mathrm{mg} \mathrm{ml}^{-1}\right)$ with or without chloroquine $\left(10 \mu \mathrm{g} \mathrm{ml}^{-1}\right)$ or TG2-gliadin peptide $\left(5 \mu \mathrm{g} \mathrm{ml}^{-1}\right)$. II27p28 (c) and Cd11b (d) expression relative to Cyclophilin were determined by real-time PCR analysis. Horizontal bars represent mean values \pm s.e.m. ( $n=5-13$ per group). Student's $t$-tests or one-way analysis of variance followed by Bonferroni post-hoc test. NS, not significant. 
underwent approximately five divisions irrespective of the nature of the APCs (Figure 4a). However, macrophages cultured with CT-TG2-gliadin consistently induced higher percentages of IFN- $\gamma^{+} \mathrm{IL}-10^{-}$-single-producing and IFN$\gamma^{+} \mathrm{IL}-10^{+}$-double-producing gliadin-specific $\mathrm{T}$ cells when compared with DCs alone. Mixtures of DC and macrophage a

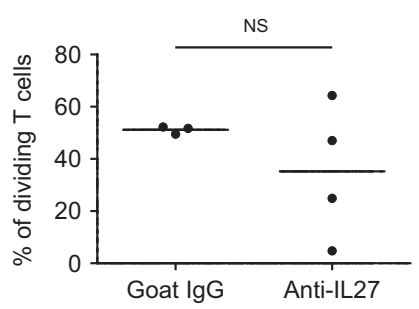

b

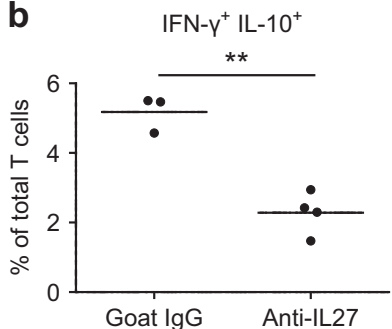

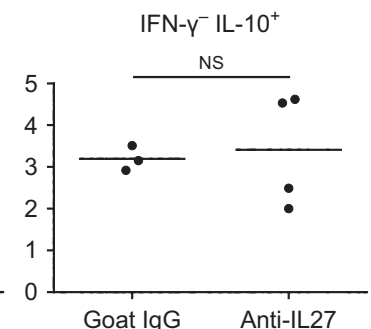

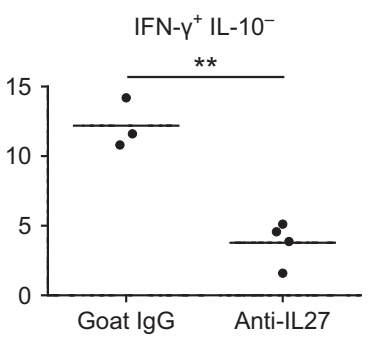

C

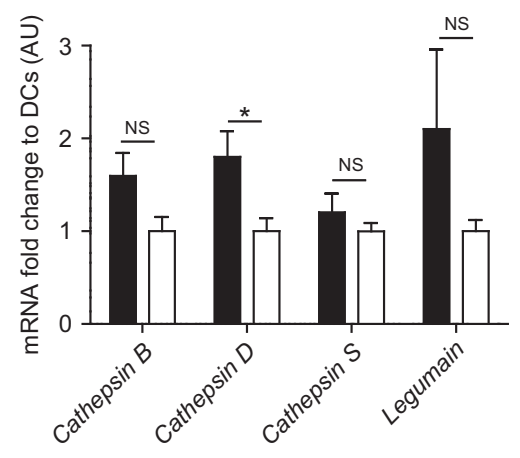

e

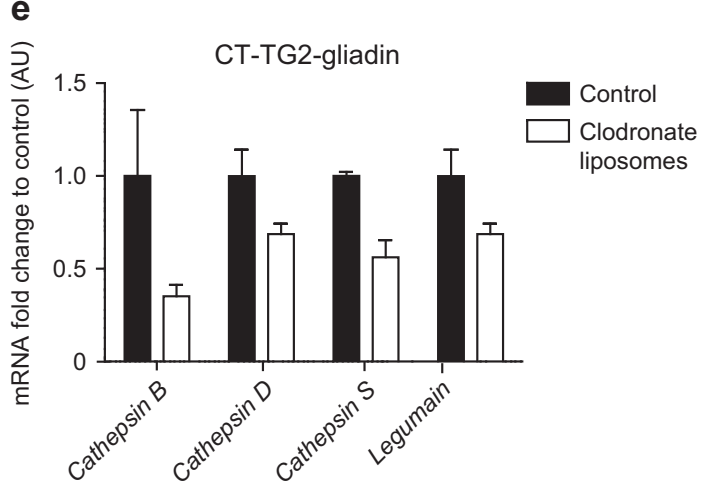

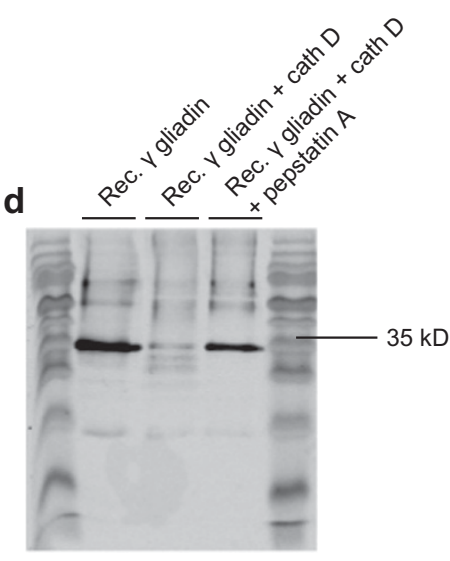

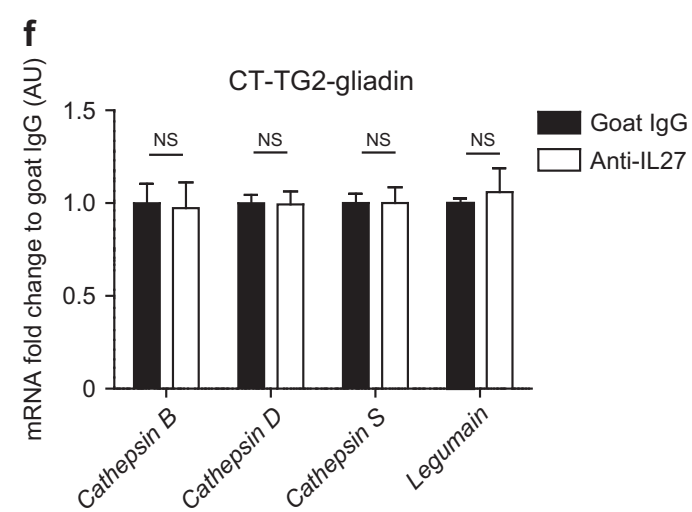

Figure 6 Interleukin (IL)-27 and Cathepsin D contribute to differentiation of IL-10 and interferon (IFN)- $\gamma$ double producing gliadin-specific T cells. (a, b) DQ2 mice received $1 \times 10^{7}$ carboxyfluorescein succinimidyl ester (CFSE)-labeled CD4 ${ }^{+}$gliadin-TCRtg T cells. Two hours prior to the T-cell transfer, mice were intraperitoneally injected with a polyclonal goat anti-IL27 antibody (Ab) (40 $\mu$ g per mouse) or control polyclonal goat immunoglobulin G (IgG) Ab ( $40 \mu \mathrm{g}$ per mouse). The day after transfer, mice received $75 \mathrm{mg}$ CT-TG2-gliadin distributed over three intragastric gavages on the same day. At $72 \mathrm{~h}$ after feed, the spleens were isolated, a fragment was stored for mRNA isolation while the remaining spleen was digested to a single-cell suspension for flow cytometric analysis. (a) Percentage of proliferating T cells gated on $\mathrm{CD}^{+}{ }^{+}$cells. (b) Percentage of IFN- $\gamma$ - and IL-10-producing cells of total $\mathrm{CFSE}^{+} \mathrm{CD} 4{ }^{+} \mathrm{V} \beta 1^{+} \mathrm{T}$ cells. Horizontal bars represent mean values. (c) Splenic macrophages or dendritic cells (DCs) from DQ2 mice were cultured for $24 \mathrm{~h}$, and the expression of the lysosomal proteases Cathepsin B, Cathepsin D, Cathepsin S, and Legumain relative to Cyclophilin was assessed by realtime PCR analysis. Fold change of expression in macrophages relative to DCs is depicted. Data displayed as mean values \pm s.e.m. ( $n=6-7$ per group) (d) Recombinant $\gamma$-gliadin was incubated with recombinant mouse Cathepsin D in the presence or absence of Pepstatin A $\left(10 \mu \mathrm{g} \mathrm{ml}{ }^{-1}\right)$. Degradation of the recombinant $\gamma$-gliadin was assessed by western blotting using an anti-gliadin primary antibody. (e) DQ2 mice received $1 \times 10^{7}$ CFSE-labeled CD4 ${ }^{+}$ gliadin-TCRtg T cells. At $24 \mathrm{~h}$ prior to the T-cell transfer, mice were either injected with $200 \mu \mathrm{l}$ clodronate liposomes or saline (control). The day after transfer, mice received $75 \mathrm{mg} \mathrm{CT-TG2-gliadin} \mathrm{distributed} \mathrm{over} \mathrm{three} \mathrm{intragastric} \mathrm{gavages} \mathrm{on} \mathrm{the} \mathrm{same} \mathrm{day.} \mathrm{At} 72 \mathrm{~h}$ after the first feed, a piece of spleen was isolated. mRNA was extracted from the splenic fragment and CDNA synthesis was performed. Expression levels of the lysosomal proteases Cathepsin B, Cathepsin D, Cathepsin S, and Legumain relative to Cyclophilin were assessed by real-time PCR analysis. The fold change of expression relative to control mice is depicted. Data are displayed as mean values \pm s.d. ( $n=2-3$ per group). (f) Splenic expression of the lysosomal proteases Cathepsin B, Cathepsin D, Cathepsin S, and Legumain relative to Cyclophilin in the experiment described in $\mathbf{a}$ and $\mathbf{b}$ was assessed by real-time PCR analysis ( $n=4$ per group). Fold change relative to values in goat IgG-treated control mice is depicted. Student's $t$-test. NS, not significant. 
cultures improved the proliferative capacity when compared with macrophages alone, but the percentage of differentiating IFN- $\gamma$ - and IL-10-double-producing cells were lower than in the cultures stimulated with CT-TG2-gliadin and macrophages only (Figure 4a,b).

These findings show that macrophages rather than DCs favor the differentiation of Tr1-like cells in response to CTTG2-gliadin in vitro.

\section{IL-27 induces IL-10 and IFN- $\gamma$ production by gliadin-specific $\mathrm{T}$ cells in vitro}

The innate immune cell-derived cytokine IL-27 has been reported to promote the induction of $\operatorname{Tr} 1$ cells. ${ }^{17,21}$ Therefore, we assessed whether recombinant IL-27 could induce Tr1-likecell differentiation in response to CT-TG2-gliadin-loaded macrophages or DCs. In CT-TG2-gliadin-loaded macrophage co-cultures, addition of exogenous IL-27 led to a threefold increase in the frequency of IFN- $\gamma$ - and IL-10-doubleproducing Tr1-like cells. In CT-TG2-gliadin-loaded DC cocultures, exogenous IL-27 also potentiated the frequency of IFN- $\gamma$ - and IL-10-double-producing Tr1-like cells, however, to a much lesser extent (Figure 5a,b). Addition of IL-27 also led to a fourfold increase in the percentage of IFN- $\gamma^{+} \mathrm{IL}-10^{-}$-singleproducing gliadin-specific T cells in CT-TG2-gliadin-loaded macrophage co-cultures. This effect of IL-27 on the induction of IFN- $\gamma$-producing gliadin-specific T cells was also less potent in CT-TG2-gliadin-loaded DC co-cultures (Figure 5a,b). In order to determine whether preferential induction of IFN- $\gamma$ and IL-10-producing Tr1-like cells by macrophages in response to CT-TG2-gliadin relied on the expression of IL-27, we next assessed the expression levels of Il27p28 in macrophages and DCs incubated with CT-TG2-gliadin. Overnight incubation of splenic macrophages with CT-TG2-gliadin induced increased expression of $1127 p 28$ as compared with medium or macrophages incubated with TG2-gliadin peptide. Moreover, Il27p28 mRNA expression was significantly higher in macrophages incubated with CT-TG2-gliadin than DCs incubated with CT-TG2-gliadin (Figure 5c). In order to establish whether Il27p28 mRNA expression correlated with macrophage activation, mRNA expression of the CR3 receptor beta integrin $C d 11 b$, having a role in adhesionmediated phagocytosis, ${ }^{22}$ was assessed. As observed for Il27p28 expression, macrophages incubated with CT-TG2-gliadin overnight expressed significantly more $C d 11 b$ mRNA than DCs incubated with CT-TG2-gliadin (Figure 5d). The increased Il27p28 did not appear to be due to endotoxin contamination, as the CT-TG2-gliadin culture did not induce increased Il12p40 expression in DCs when compared with medium alone nor did neutralization of TLR4 signaling abrogate the CT-TG2-gliadin-induced Il27p28 expression in splenic macrophages (data not shown). Finally, as Il27p28 expression was specifically induced in macrophages incubated with CT-TG2-gliadin but not TG2-gliadin peptide, we hypothesized that induction of Il27p28 expression occurred consequently to gliadin uptake and degradation. To test this, the protease inhibitor chloroquine was added concomitantly with CT-TG2-gliadin to the macrophages. As anticipated, chloroquine treatment fully abolished the CT-TG2-gliadininduced increase in $I 127 p 28$ expression previously observed in macrophages (Figure 5c) and decreased the expression of the cellular activation molecule $C d 11 b$ (data not shown). This was not due to a general effect of chloroquine on the translation of all cytokine genes as the expression level of Tnfa, Il10, and Il6 was not significantly affected by chloroquine treatment in macrophages incubated with CT-TG2-gliadin (see Supplementary Figure S2a). Similarly, the effect of chloroquine was not due to increased cell death as chloroquine treatment did not increase apoptosis as reflected by the $B a x / B c l 2$ expression ratio (respectively, a pro-apoptotic and antiapoptotic molecule), or the $\mathrm{Ct}$ values of the reference gene Cyclophilin (see Supplementary Figure S2b).

In conclusion, expression of Il27p28, a factor that drives Tr1-cell differentiation, is specifically induced in macrophages as a consequence of CT-TG2-gliadin uptake and degradation and addition of IL-27 potentiates gliadin-specific Tr1-like-cell differentiation in vitro with a more potent effect on $\mathrm{T}$ cells co-cultured with CT-TG2-gliadin-loaded macrophages than DCs.

\section{IL-27 and Cathepsin D contribute to differentiation of IL-10- and IFN- $\gamma$-double-producing gliadin-specific $\mathrm{T}$ cells}

To demonstrate that IL-27 drives differentiation of gliadinspecific IL-10- and IFN- $\gamma$-producing T cells in vivo, DQ2 mice were treated with a neutralizing anti-IL27 or control immunoglobulin $\mathrm{G}$ (IgG) Ab $2 \mathrm{~h}$ prior to $\mathrm{CD} 4{ }^{+}$gliadin-TCRtg $\mathrm{T}$-cell transfer and subsequent CT-TG2-gliadin feed. At $72 \mathrm{~h}$ after gliadin feed, neutralization of IL-27 reduced proliferation of splenic gliadin-specific $\mathrm{T}$ cells in some but not all mice (Figure 6a). The treatment significantly reduced the percentage of IFN- $\gamma^{+} \mathrm{IL}-10^{+}$and IFN- $\gamma^{+}$IL- $10^{-}$gliadin-specific T cells (Figure 6b), thereby establishing a role for IL-27 in differentiation of splenic Tr1-like gliadin-specific $\mathrm{T}$ cells after gliadin feed. Recently, it has been reported that differentiation of human monocyte-derived DCs in the presence of IL-27 drives maturation of the pro-enzyme of Cathepsin D into the mature active form. ${ }^{16}$ Cathepsin D, a major component of lysosomes, is a highly active endopeptidase with optimal activity in an acid ( $\mathrm{pH} 3-5)$ environment, preferentially cleaving peptide bonds that are flanked by large hydrophobic amino acids, and is inhibited by pepstatin A. ${ }^{23}$ This led us to assess whether splenic macrophages and DCs from DQ2 mice differ in the expression of particular endopeptidases among which is Cathepsin D. Indeed, freshly isolated splenic macrophages from DQ2 mice had a significantly higher expression of Cathepsin D mRNA relative to splenic DCs (Figure 6c). To determine whether this increased expression could have relevance for gliadin degradation, we assessed whether gliadin is a substrate for Cathepsin D. Thereto recombinant $\gamma$-gliadin, which contains the DQ2.5-glia- $\gamma 1$ epitope (Uniprot no. AJ416339; $32 \mathrm{kD}$ ), was generated and incubated for $1 \mathrm{~h}$ with recombinant Cathepsin $\mathrm{D}$ in the presence or absence of pepstatin A. The preparations were analyzed by western blotting using a polyclonal anti-gliadin $\mathrm{Ab}$. 
As can be seen in Figure 6d, incubation of recombinant $\gamma$-gliadin with Cathepsin D elicited degradation of the $32-\mathrm{kD}$ band (lane 2); a process that was inhibited upon co-incubation with pepstatin A (lane 3). Specificity of the western blotting was confirmed by analysis of sodium dodecyl sulfatepolyacrylamide gel electrophoresis (SDS-PAGE) separated bands using chymotrypsin digestion and subsequent mass spectrometry identifying the $32-\mathrm{kD}$ band as $\gamma$-gliadin (data not shown). These data would argue that macrophages containing lysosomes with increased amounts of Cathepsin D would have enhanced capacity to digest gliadin and drive differentiation of gliadin-specific Tr1-like cells in vivo. Theoretically, IL-27 could enhance this effect by posttranscriptional modification of the pro-enzyme Cathepsin D into its mature form. Unfortunately, we currently have no means to selectively increase Cathepsin D activity in macrophages in vivo to demonstrate this. However, at $72 \mathrm{~h}$ after CT-TG2-gliadin feed, the spleens of macrophagedepleted DQ2 mice exhibited reduced mRNA expression of multiple endopeptidases (Figure 6e) but not when IL-27 was neutralized in vivo, showing that downregulation of the endopeptidase level is specific to macrophage depletion (Figure 6f).

\section{DISCUSSION}

In this study, we report that macrophages are crucial for the differentiation of gliadin-specific Tr1-like cells in vivo. We previously showed that these Tr1-like cells mediate tolerance to gliadin in DQ2.gliadinTCR mice. ${ }^{10}$ Although splenic DCs have the capacity to elicit gliadin-specific $\mathrm{T}$-cell proliferation in vitro and in vivo, these cells did not induce Tr1-like-cell differentiation as effectively as splenic macrophages. Macrophages have increased capacity for lysosomal proteolysis when compared with DCs. ${ }^{15}$ Upon comparison of splenic macrophages and DCs, macrophages expressed increased amounts of Cathepsin $\mathrm{D}$, an endopeptidase which degraded recombinant gliadin in vitro. In agreement, depletion of macrophages in vivo reduced splenic Cathepsin D expression and inhibited the splenic Tr1-like-cell response to oral CT-TG2-gliadin while macrophage depletion did not alter the splenic $\mathrm{T}$-cell response to TG2-gliadin peptide feed. Upon incubation with CT-TG2gliadin but not TG2-gliadin peptide, macrophages expressed Il27p28, a factor that has been shown to increase Cathepsin D activity and mediate gliadin-specific Tr1-like cell differentiation in vivo. DCs did not upregulate the expression of Il27p28 upon CT-TG2-gliadin incubation. Together, these data reveal an important role for macrophages in the development of gliadin-specific Treg cell responses.

Previously, we have shown that, in mice, oral tolerance to gliadin is mechanistically different from oral tolerance to the soluble food protein OVA. Although OVA feed elicits the conversion of naive $\mathrm{T}$ cells into suppressive Foxp $3^{+}$Treg cells in the gut-draining MLNs, oral gliadin induces differentiation of suppressive IL-10-secreting Tr1-like cells in the spleen. ${ }^{10}$ The unexpected finding that an immune response to a food protein was mounted in the spleen rather than in a gut-draining lymphoid tissue and the unusual molecular characteristics of deamidated gliadin led us to hypothesize that degradation of gliadin is a pivotal step in the differentiation of Tr1-like cells and the subsequent development of oral tolerance to gliadin. Therefore, we first aimed to dissect whether insufficient degradation and presentation of gliadin may contribute to the suboptimal development of a T-cell response in the MLNs after gliadin feed. As expected using immunohistochemistry and feeding fluorescently-labeled CT-TG2-gliadin, the labeled protein was detectable in $\mathrm{CD} 103^{+} \mathrm{CD} 11 \mathrm{~b}^{-}$APCs within the MLNs showing that the antigen could drain to the gut-draining lymphoid tissue but did not induce a robust $\mathrm{T}$-cell response. However, productive T-cell responses in the MLNs could be obtained after feeding TG2-gliadin peptide, suggesting that extracellular MHCII loading could impose gliadin presentation in the MLNs after gliadin feed. Dietary gluten contains a number of epitopes that were identified as T-cell receptor (TCR) binding in CD patients. ${ }^{24}$ The common feature of these epitopes is the presence of high levels of proline and glutamine residues, making the gluten protein resistant to degradation., ${ }^{2,25}$ Macrophages, and particularly those in the splenic marginal zone, are equipped with high amounts of lysosomal proteases such as Cathepsin S, Cathepsin B, and Cathepsin D, whereas other APCs such as DCs and B cells express these to a lower degree. ${ }^{15}$ Therefore, we envisaged that preferential splenic Tr1-like-cell differentiation after CT-TG2-gliadin feed involved macrophages and have proven that these cells have a pivotal role in mounting gliadin-specific Tr1-like differentiation in the spleen.

To demonstrate that differentiation of splenic Tr1-like cells after CT-TG2-gliadin feed is dependent on macrophages, we depleted macrophages in vivo by i.v. injecting clodronate liposomes into mice. As shown previously, this technique induces apoptosis of CD169-positive marginal zone metallophilic macrophages that are situated at the border of follicles and the marginal zone inducing a cellular depletion up to $48 \mathrm{~h}$ after injection. ${ }^{19}$ Crucially, macrophage depletion dramatically reduced the differentiation of Tr1-like cells after CT-TG2gliadin feed, arguing that macrophages may be pivotal for the tolerogenic Tr1-like-cell response to gliadin. Intriguingly, the clodronate liposome treatment did not abolish proliferation of T cells. Possibly, remaining splenic DCs have the capacity to induce this proliferation but lack the capacity to drive T-cell differentiation.

Our results show that gliadin presentation leading to $\mathrm{T}$-cell proliferation can be performed by APCs other than macrophages, but macrophages are essential for providing the qualitative costimulatory signals leading to the differentiation of gliadin-specific IFN- $\gamma$ - and IL-10-secreting Tr1-like cells after presentation. However, owing to technical difficulties in purifying the macrophage subpopulations in the MLNs, we have not yet been able to resolve whether macrophageindependent uptake, insufficient numbers of macrophages, reduced endopeptidase activity, or reduced IL-27 secretion account for the suboptimal Tr1-like induction in the MLNs. Baseline endopeptidase mRNA levels in whole MLNs of DQ2 mice were equally high to baseline endopeptidase mRNA levels 
in the spleen (data not shown), possibly suggesting that a reduced expression of lysosomal endopeptidases is not the underlying cause of the absence of T-cell proliferation observed in the MLNs.

We have previously shown that these Tr1-like gliadinspecific $\mathrm{T}$ cells generated in the spleen in our model are tolerogenic and able to suppress inflammatory $\mathrm{T}$-cell responses by inhibiting IFN- $\gamma$ production in an IL-10-dependent manner. ${ }^{10}$ In this previous as well as in the present study, gliadin-specific $\operatorname{Tr} 1$-like cells do secrete relatively large amounts of IFN- $\gamma$ simultaneously to the production of IL10. Furthermore, a substantial percentage of gliadin-specific $T$ cells do not produce IL-10 but only secrete IFN- $\gamma$, and in vivo macrophage depletion or neutralization of IL-27 also inhibited differentiation of this IFN- $\gamma$-single-producing subpopulation. In this mixed population, these IFN- $\gamma$-producing cells, whose phenotype resembles $\mathrm{T}$ helper 1 cells, are not sufficient to drive an inflammatory T-cell response, as the total pool of gliadinspecific $T$ cells generated in our model exerts suppressive effects when adoptively transferred. ${ }^{10}$ Possibly, these IFN- $\gamma$-producing gliadin-specific $\mathrm{T}$ cells could constitute an intermediary stage of Tr1-like cells susceptible to start producing IL-10, for example, consequently to the repetitive stimulation of their TCR. ${ }^{26,27}$

Recent reports have identified IL-27 as a driving factor for the induction of IL-10-producing Tr1 cells in mouse and human. ${ }^{17}$ IL-27 and its target transcription factor c-Maf, the cytokine IL-21, and the costimulatory receptor inducible T-cell co-stimulator (ICOS) coordinately act together to promote Tr1-cell differentiation. ${ }^{17}$ Mice deficient for either c-Maf, IL-21, or ICOS have decreased capacity for IL-27-induced differentiation of $\operatorname{Tr} 1$ cells. ${ }^{17}$ In our in vitro experiments, macrophages cultured with CT-TG2-gliadin expressed high levels of Il27p28 mRNA in comparison to DCs cultured with CT-TG2-gliadin, whereas TG2-gliadin peptide did not induce Il27p28 mRNA expression in macrophages. Strikingly, treatment with the protease inhibitor chloroquine abolished the increase in Il27p28 expression in macrophages in response to CT-TG2-gliadin. These results may suggest that degradation of the CT-TG2-gliadin by macrophages contributes to IL-27 induction. This preferential expression of IL-27 by splenic macrophages has previously been reported in other settings. In patients with human visceral leishmaniasis, splenic $\operatorname{Tr} 1$ cells are an important source of IL-10. These patients present elevated plasma levels of IL-27 and splenic CD14 ${ }^{+}$macrophages were shown to be the main expressors of Il27p28. ${ }^{28}$ Strikingly, addition of recombinant IL-27 to our in vitro cultures drastically potentiated the percentage of IFN- $\gamma$ - and IL-10secreting gliadin-specific Tr1-like cells, but the effect was more potent in co-culture of T cells with macrophages than with DCs, suggesting that IL-27 induces Tr1-cell differentiation not only by acting on $\mathrm{T}$ cells but also on macrophages.

In a recent report, IL-27 has been reported to enhance activation of the endopeptidase Cathepsin D in differentiation cultures of DCs. ${ }^{16}$ In addition, Cathepsin $D$ is differentially expressed in DQ2 mouse macrophages when compared with DCs and its expression is reduced in the spleens of macrophage- depleted mice but not anti-IL27-treated mice. Our in vitro data show that recombinant $\gamma$ gliadin is readily cleaved by Cathepsin $\mathrm{D}$, a process inhibited by pepstatin $\mathrm{A}$, an inhibitor of both Cathepsin D and Cathepsin E. These data suggest that Cathepsin D, a major lysosomal protease in macrophages, may have the capacity to contribute to degradation of partially digested gliadin. Further detailed analysis should focus on identifying the contribution of this enzyme compared with other lysosomal proteases and assess whether its function contributes to gliadin-specific Tr1-like differentiation.

In summary, the results obtained in this study support a role for macrophages in the induction of tolerance to gluten. Macrophages are of crucial importance to drive degradation of gliadin, possibly involving Cathepsin $\mathrm{D}$, and provide the cytokine signal IL-27 favoring the differentiation of gliadinspecific Tr1-like cells in the spleen. Our work therefore raises the question whether loss of tolerance to gluten in $\mathrm{CD}$ might be related to defective macrophage-induced regulation. Currently, it is still unclear whether changes in Tr1-like cell function contribute to human $\mathrm{CD}$ as these cells have no specific surface marker making their quantification and functional analysis difficult. In pediatric CD patients, IL-10 release has been observed in isolated intestinal T-cell populations from patients who had been challenged with a gluten-containing diet. ${ }^{29}$ Moreover, it has previously been shown that suppressive IL-10producing gliadin-specific $\mathrm{T}$-cell clones can be cultured from the mucosa of treated CD patients ${ }^{30}$ and is upregulated in potential $\mathrm{CD}$ patients with a positive $\mathrm{CD}$-associated serology but Marsh 0 intestinal histology score. ${ }^{31}$ As Foxp $3^{+}$Tregs also have the capacity to secrete IL-10, and alterations in effector T-cell sensitivity to suppression by this population have been described in $C D$ patients, ${ }^{32}$ further studies are required to dissect possible roles of these two Treg cell populations in mediating tolerance to gliadin.

\section{METHODS}

Mice. HLA-DQ2 $\left(\mathrm{DQA1}^{\star} 0501, \quad \mathrm{DQB1}{ }^{\star} 0201=\mathrm{HLADQ} 2.5\right)$ $\mathrm{MHCII}^{\Delta / \Delta}$ transgenic (DQ2) mice and gliadin-TCR.MHCII ${ }^{\Delta / \Delta}$ transgenic (gliadinTCR) mice, which have a transgenic TCR specific for the DQ2.5-glia- $\gamma 1$ epitope were bred at the Erasmus MC. Singletransgenic DQ2 and gliadinTCR mice were maintained on a C57BL/6DBA/2 mixed background. HLADQ2.gliadin-TCR.MHCII ${ }^{\Delta / \Delta}$ double transgenic (DQ2.gliadinTCR) mice were obtained by crossing DQ2 mice with gliadinTCR mice. All mice were kept under specific pathogen-free housing conditions and the animal experimental committee of the Erasmus Medical Center approved the experiments. DQ2 mice, gliadinTCR mice, and DQ2.gliadinTCR mice were bred and maintained on a gluten-free chow (Arie Blok BV, Woerden, The Netherlands).

Proteins and peptides. Crude gliadin from wheat (Sigma Aldrich, Zwijndrecht, The Netherlands) was dissolved in $0.1 \mathrm{M} \mathrm{NH}_{4} \mathrm{HCO}_{3}$ and $2 \mathrm{M}$ urea buffer $\left(100 \mathrm{mg} \mathrm{ml}^{-1}\right)$ and digested with $50 \mu \mathrm{g} \mathrm{ml}^{-1}$ $\alpha$-chymotrypsin (Sigma) at room temperature for $24 \mathrm{~h}$. Digestion was stopped by heating to $98^{\circ} \mathrm{C}$ for $10 \mathrm{~min}$. The chymotrypsin-digested gliadin (CT-gliadin) was centrifuged $(5000 \mathrm{~g}, 45 \mathrm{~min})$, filter-sterilized $(0.45 \mu \mathrm{m})$ and dialyzed against sterile phosphate-buffered saline. Protein concentration was determined using a bicinchoninic acid assay (Perbio Science, Etten-Leur, The Netherlands). To obtain chymotrypsin-digested deamidated gliadin (termed CT-TG2-gliadin), 
CT-gliadin was treated with guinea pig liver transglutaminase (TG2; $0.08 \mathrm{U} \mathrm{mg}^{-1}$; Zedira $\mathrm{GmbH}$, Darmstadt, Germany) for $16 \mathrm{~h}$ at $37^{\circ} \mathrm{C}$. For in vivo experiments, mice received 60 or $75 \mathrm{mg}$ CT-TG2-gliadin distributed over three intragastric gavages (i.g.) on one single day or received $3 \mathrm{mg}$ of the synthetic TG2-gliadin- $\gamma 1$-E peptide (termed TG2gliadin peptide) (QPEQPQQSFPEQERPF) per day on 3 consecutive days by i.g. For in vitro experiments, CT-TG2-gliadin $\left(0.5 \mathrm{mg} \mathrm{ml}^{-1}\right)$ or the TG2-gliadin peptide $\left(5 \mu \mathrm{g} \mathrm{ml}^{-1}\right)$ was used.

Recombinant $\gamma$-gliadin expression. Competent BL21 (DE3) pLysS were transformed with a pET14b_29-35 $\gamma 5$ gliadin plasmid generated in the laboratory of L.M. Sollid. Bacteria were grown in Luria Broth medium to an optical density at $600 \mathrm{~nm}$. Protein expression was induced by adding $0.4 \mathrm{~mm}$ of isopropyl thiogalactose, and bacteria were grown overnight at $37^{\circ} \mathrm{C}$ while shaking. Bacteria were harvested by centrifugation and the pellet was lysed in $300 \mathrm{~mm} \mathrm{NaCl}, 50 \mathrm{~mm} \mathrm{NaP}-$ buffer, $10 \mathrm{~mm}$ imidazole, and $\mathrm{pH} 8$-containing protease inhibitors (phenylmethanesulfonylfluoride $1 \mathrm{~mm}$, Leupeptin $1 \mu \mathrm{g} \mathrm{ml}^{-1}$, and Aprotinin 1:1000). Lysozyme was added at a final concentration of $1 \mathrm{mg} \mathrm{ml}^{-1}$, and the lysate was incubated for $30 \mathrm{~min}$ on a rotor. After sonication and centrifugation, the pellet was resuspended overnight in a buffer containing $8 \mathrm{M}$ urea, $300 \mathrm{~mm} \mathrm{Nacl,} 50 \mathrm{~mm}$ NaP-buffer, and $10 \mathrm{~mm}$ imidazole, pH8. After centrifugation, the supernatant was purified using on a Ni-NTA column (Qiagen, Venlo, The Netherlands). Protein was eluted from the column with a buffer containing $8 \mathrm{~m}$ urea, $300 \mathrm{~mm} \mathrm{NaCl}, 50 \mathrm{~mm}$ NaP-buffer, and $250 \mathrm{~mm}$ imidazole. The eluate was dialyzed against a buffer containing $0.1 \mathrm{M} \mathrm{NaH}_{4} \mathrm{HCO}_{3}$ and $2 \mathrm{~m}$ urea using a dialysis membrane with a $12-14-\mathrm{kDa}$ molecular weight cutoff. Total protein concentration was determined using a bicinchoninic acid assay (Thermo Scientific, Fremont, CA) according to the manufacturer's protocol.

Recombinant $\gamma$-gliadin degradation by Cathepsin D and antigliadin western blotting. Degradation of recombinant $\gamma$-gliadin protein by Cathepsin D was performed as follows. Cathepsin D (R\&D Systems, Minneapolis, MN) was diluted to $20 \mu \mathrm{g} \mathrm{ml}^{-1}$ in assay buffer (100 mm NaAc, $200 \mathrm{~mm} \mathrm{NaCl}, \mathrm{pH} 3.5)$ and incubated at room temperature for $10 \mathrm{~min}$. Cathepsin $\mathrm{D}$ was added to the recombinant $\gamma$-gliadin $\left(118 \mu \mathrm{g} \mathrm{ml}^{-1}\right)$ in a 1 -to-1 ratio. The cathepsin D/recombinant $\gamma$-gliadin mix was incubated for $1 \mathrm{~h}$ at $37^{\circ} \mathrm{C}$ in the presence or absence of pepstatin A $\left(10 \mu \mathrm{g} \mathrm{ml}^{-1}\right)$ (Sigma). To detect the nondegraded form of recombinant $\gamma$-gliadin, the Cathepsin D/recombinant $\gamma$-gliadin ( \pm pepstatin A) mix was loaded and run on a $15 \%$ SDS-PAGE gel. The gel was blotted at $100 \mathrm{~V}$ on a $0.22-\mu \mathrm{m}$ nitrocellulose membrane. The membrane was blocked in blocking buffer (TBS, 5\% milk powder, $0.1 \%$ Tween-20) and then incubated with a rabbit anti-gliadin primary $\mathrm{Ab}$ (Antibodies online, Aachen, Germany), followed by an incubation with a goat anti-rabbit IgG secondary Ab (Li-Cor, Bad-Homburg, Germany). Positivity was visualized using an Odyssey imaging system (Li-Cor).

Tracking of CT-TG2-gliadin. DQ2 mice received $3.2 \mathrm{mg}$ CT-TG2gliadin intragastrically or $1 \mathrm{mg}$ i.v. At $18 \mathrm{~h}$, spleens and MLNs were isolated and embedded in cryopreservative solution (optimal cutting temperature, Tissue-Tek, Miles, Elkhart, IN). Immunohistochemical stainings were performed on $6-\mu \mathrm{m}$ cryostat sections. Prior to staining, the samples were fixed with ice-cold acetone for $10 \mathrm{~min}$ and blocked with $10 \%$ normal mouse serum and $10 \%$ normal goat serum. Tissue sections were incubated using anti-gliadin primary $\mathrm{Ab}$ (Cusabio, rabbit polyclonal) and subsequently stained with secondary Ab goatanti-rabbit fluorescently labeled with DyLight-488 (Thermo Scientific). Control stainings were performed using no anti-gliadin Ab. Sections were mounted with medium for fluorescence containing 4,6diamidino-2-phenylindole (Vector Laboratories, Burlingame, CA). A conjugate control was performed for each immunostaining. Images were acquired and analyzed using a Leica DM5500B upright microscope and LAS-AF image acquisition software (Leica Microsystems, Rijswijk, The Netherlands).
For flow cytometry, CT-TG2-gliadin was labeled with Alexa Fluor 647 succinimidyl ester according to the manufacturer's protocol (Invitrogen, Breda, The Netherlands). DQ2 mice received $3.2 \mathrm{mg}$ Alexa Fluor 647-labeled CT-TG2-gliadin by by intragastric gavage or $1.0 \mathrm{mg}$ Alexa Fluor 647 labeled CT-TG2-gliadin intravenously. Control mice received the same amount of non-labeled CT-TG2gliadin by gavage. At $18 \mathrm{~h}$ after gavage, MLNs were isolated and singlecell suspensions were prepared by using Liberase TM (Roche, Woerden, The Netherlands) and DNAse (Sigma). After staining (see "Flow cytometry" below), cells were analyzed by flow cytometric analysis for detection of Alexa Fluor 647-positive cells.

Adoptive transfer. Lymph nodes and spleens were obtained from DQ2.gliadinTCR mice. After erythrocyte lysis, the single-cell suspension was enriched for $\mathrm{CD}^{+}{ }^{+}$cells by depletion of $\mathrm{B}$ cells, macrophages, monocytes, and $\mathrm{CD} 8{ }^{+}$cells with rat Abs against $\mathrm{B} 220$ (clone 6B2), F4/80 (BM8), CD11b (MAC-1), MAC-2, MHCII (M5/114) and CD8 (53.6.72) and anti-rat magnetic Dynabeads (Invitrogen). The enriched cells were fluorescently labeled with CFSE (Molecular Probes, Leiden, The Netherlands) at a final concentration of $5 \mu \mathrm{M}$ to follow their division profile. DQ2 acceptor mice received $1 \times 10^{7}$ CFSE-labeled CD $4^{+}$gliadin-TCRtg T cells by i.v. injection in the tail vein. One day after transfer, DQ2 mice received 60 or $75 \mathrm{mg}$ CT-TG2-gliadin distributed over three i.g. on the same day or received i.g. TG2-gliadin peptide $3 \mathrm{mg}$ day $^{-1}$ on 3 consecutive days. At $72 \mathrm{~h}$ after the first feed, MLNs and spleen were isolated and examined for the division of transferred $\mathrm{CD}^{+}{ }^{+}$gliadin-TCRtg T cells.

Clodronate liposome and anti-IL27 treatment. Clodronate liposomes were prepared as described previously. ${ }^{20}$ To deplete macrophages, DQ2 mice were injected i.v. with $200 \mu \mathrm{l}$ liposomes or saline as control. Polyclonal goat anti-IL27 (R\&D Systems) (40 $\mu$ g per mouse) was administered intraperitoneally $2 \mathrm{~h}$ prior T-cell transfer. Polyclonal goat IgG (R\&D Systems; $40 \mu \mathrm{g}$ per mouse) was administered intraperitoneally as an isotype control.

In vitro restimulation. For in vitro restimulation experiments, cells were purified from spleens or MLNs of DQ2 acceptor mice. Next, $5 \times 10^{5} \mathrm{CD}^{+}$gliadin-TCRtg $\mathrm{T}$ cells were restimulated with $5 \mu \mathrm{g} \mathrm{ml}^{-1} \mathrm{TG} 2$-gliadin peptide for $24 \mathrm{~h}$. For intracellular detection of cytokines, cells were restimulated with $0.05 \mu \mathrm{g} \mathrm{ml}^{-1}$ phorbol 12 -myristate 13 -acetate (Sigma) and $0.1 \mu \mathrm{g} \mathrm{ml}^{-1}$ ionomycin (Sigma) and Monensin (Golgistop; BD, Breda, The Netherlands) for $4 \mathrm{~h}$ of culture.

Flow cytometry. The following Abs were used for flow cytometry: 7AAD (7-aminoactinomycin D; Molecular Probes), anti-CD3 (BD, clone 145-2C11), anti-CD4 (BD, clone RM4-5), anti-CD45 (Invitrogen; clone 30-F11), anti-CD8 $\alpha$ (BD, clone 53-6.7), anti-CD11c (Biolegend, clone HL3), anti-CD103 (BD, clone M1/70), anti-CD11b (BD, clone M1/70), B220 (Biolegend, San Diego, CA, clone RA3-6B2), and anti-TCR V $\beta 1$ (Beckman Coulter, Woerden, The Netherlands; clone BL37.2). Anti-HLA-DQ (SPV-L3) was kindly provided by Dr. H. Spits, Amsterdam, The Netherlands.

Intracellular staining for Foxp3 (eBiosciences, Bergen op Zoom, The Netherlands; clone FJK-16S), anti-IL-10 (eBiosciences clone JES5-16E3), and anti-IFN- $\gamma$ (eBiosciences; clone XMG1.2) or the appropriate isotype controls was performed with the Foxp3 Staining Buffer Kit, according to manufacturer's protocol (eBiosciences). Phenotype and cell division were measured on a FACSCanto II (BD). Data were analyzed using the FlowJo software (Flowjo, Ashland, OR).

Immunohistochemistry. At $72 \mathrm{~h}$ after adoptive $\mathrm{CD} 4^{+}$gliadin-TCRtg T-cell transfer and gliadin feed as described above, spleens of DQ2 acceptor mice were isolated. Prior to staining, the samples were fixed with ice-cold acetone for $10 \mathrm{~min}$ and blocked with $10 \%$ normal mouse serum. Tissue sections were incubated using a primary $\mathrm{Ab}$ for 
anti-CD169 (Acris antibodies, Herford, Germany, clone MOMA-1) or $\mathrm{CD} 11 \mathrm{c}$ primary $\mathrm{Ab}$ (Biolegend, clone $\mathrm{N} 418$ ). Immunoreactive sites were visible by staining with $3,3^{\prime}$-diaminobenzidine tetrahydrochloride (Sigma) after CD169 staining. CD11c was subsequently stained with secondary Ab goat-anti-Armenian hamster labeled with a DyLight-594 (Thermo Scientific), and the sections were mounted with medium for fluorescence containing 4,6-diamidino-2-phenylindole (Vector Laboratories, Burlingame, CA). A conjugate control was performed for each immunostaining. Images were acquired and analyzed using a Leica DM5500B upright microscope and LAS-AF image acquisition software (Leica Microsystems).

In vitro T-cell differentiation. Spleens of DQ2 mice were isolated and single-cell suspensions were prepared. Spleens were digested using Liberase TM and DNAse. After erythrocyte lysis, CD11c ${ }^{+}$DC were isolated using anti-CD11c MACS beads (Miltenyi Biotec, Bergisch Gladbach, Germany). The negative fraction $\left(\mathrm{CD} 11 \mathrm{~b}^{+} \mathrm{CD} 11 \mathrm{c}^{\text {neg }}\right)$ after the MACS (magnetism-activated cell sorting) cell separation was plated in a 96-well tissue culture dish. After $2.5 \mathrm{~h}$ incubation to allow adherence of macrophages, monolayers were washed to remove the non-adherent cells and the adherent macrophages were incubated with medium.

$\mathrm{CD} 4{ }^{+}$gliadin-TCRtg T cells were obtained from spleens and lymph nodes from DQ2.gliadinTCR mice as described above. The enriched cells were fluorescently labeled with CFSE. A total of $2 \times 10^{4} \mathrm{DQ} 2^{+}$ cells, DCs or macrophage or both, were incubated with $5 \times 10^{5}$ CFSElabeled $\mathrm{CD} 4^{+} \mathrm{VB1} 1^{+}$gliadin-specific T cells in the presence of medium, CT-TG2-gliadin $\left(0.5 \mathrm{mg} \mathrm{ml}^{-1}\right)$, TG2-gliadin peptide $\left(5 \mu \mathrm{g} \mathrm{ml}^{-1}\right)$, IL$27\left(25 \mathrm{ng} \mathrm{ml}^{-1}\right)$ (R\&D Systems), recombinant human transforming growth factor- $\beta\left(20 \mathrm{ng} \mathrm{ml}^{-1}\right)$, or $10 \mathrm{~nm}$ retinoic acid for 24 or $72 \mathrm{~h}$ as depicted in the figure legends. Cells were analyzed using flow cytometric analysis.

Real-time PCR. Splenic macrophages $\left(\mathrm{CD} 11 b^{+} \mathrm{CD} 11 c^{\text {neg }}\right)$ or $\mathrm{DC}$ $\left(\mathrm{CD} 11 \mathrm{c}^{+}\right)$from DQ2 mice were isolated as described above. A total of $5 \times 10^{5}$ cells were incubated with CT-TG2-gliadin $\left(0.5 \mathrm{mg} \mathrm{ml}^{-1}\right)$ with or without chloroquine $\left(10 \mu \mathrm{g} \mathrm{ml}^{-1}\right)$ (Sigma) or TG2-gliadin peptide $\left(5 \mu \mathrm{g} \mathrm{ml}^{-1}\right)$. After $24 \mathrm{~h}$, cells were isolated and total RNA was purified from the cell cultures using the NucleoSpin RNA XS Kit (MacheryNagel, Düren, Germany). Up to $1 \mu \mathrm{g}$ RNA was reverse transcribed to single-stranded cDNA using a mix of random hexamers $(2.5 \mu \mathrm{M})$ and oligo(dT) primers $(20 \mathrm{~nm})$. The reverse transcription reaction was performed in a total volume of $25 \mu \mathrm{l}$ containing $0.2 \mathrm{~mm}$ of each dNTP (Amersham Pharmacia BioTech, Piscataway, NJ), $200 \mathrm{U}$ Moloney murine leukemia virus reverse transcriptase (M-MLV RT; Promega, Madison, WI) and $25 \mathrm{U}$ RNAsin (Promega). The conditions for the reverse transcription reaction were $37^{\circ} \mathrm{C}$ for $30 \mathrm{~min}, 42^{\circ} \mathrm{C}$ for $15 \mathrm{~min}$, and $94^{\circ} \mathrm{C}$ for $5 \mathrm{~min}$. The cDNA was diluted fivefold and stored at $-20^{\circ} \mathrm{C}$. Real-time quantitative PCR was performed using general fluorescence-based detection with SYBR green on an AbiPrismR 7900 Sequence Detection System (PE Applied Biosystems, Foster City, CA). Cyclophillin was used to control for sample loading and to allow normalization between samples. The expression levels relative to Cyclophillin were calculated following the equation: relative expression level $=2^{-\Delta \mathrm{Ct}}$, whereby $\Delta \mathrm{Ct}=\mathrm{Ct}_{\text {target }}-\mathrm{Ct}_{\text {cyclophillin. }}$. Specific primers were designed across different constant region exons resulting in the following primers: Cyclophillin forward $5^{\prime}$-AACCCCACCGTGTTC T-3', reverse $5^{\prime}$-CATTATGGCGTGTAAAGTCA-3'; Il27p28 forward $5^{\prime}$-CGCAGGGAATTCACAGT- ${ }^{\prime}$, reverse $5^{\prime}$ AGCGAGGAAGCA G;AGTCT-3'; Cd11b forward 5'-AAGGCTTTGGACAGAGTGT-3', reverse $5^{\prime}$-TGGGGGACAGTAGAAACA-3', Bax forward $5^{\prime}$-AAGGCCCTGTGCACTAA- ${ }^{\prime}$, reverse $5^{\prime}$ GAGGCGGTGAGGAC TC-3'; Bcl2 forward 5'-GGTGGGGTCATGTGTGT-3', reverse 5'-CTGGGGCCATATAGTTCC-3'; Cathepsin D forward 5' $5^{\prime}$ TGCA GCCAAGTTTGATG-3' ${ }^{\prime}$, reverse $5^{\prime}$-TGCCACCAAGCATTAGTT-3'; Cathepsin $B$ forward $5^{\prime}$-GGTGCCTTCACTGTGTTT-3', reverse $5^{\prime}$-CCAGCCACAATTTCTGAT-3'; Cathepsin $S$ forward 5'-CTCCG
AAGACTGTCACTTTC- $3^{\prime}$, reverse 5'-CCCCCATTATCAATGA TG-3'; and Legumain forward $5^{\prime}$-ACCGGAGAGGATGTGACT-3', reverse $5^{\prime}$-GGTCGGTGAAGTAAATGAAG-3'.

Statistics. Data are expressed as mean \pm s.d. or mean \pm s.e.m. and analyzed using Student's $t$-test or one-way analysis of variance followed by a Bonferroni post-hoc test. $P<0.05$ was considered significant. ${ }^{\star} P<0.05 ;{ }^{* *} P<0.01 ;{ }^{* *} P<0.001$.

SUPPLEMENTARY MATERIAL is linked to the online version of the paper at http://www.nature.com/mi

\section{ACKNOWLEDGMENTS}

We thank Jan Wouter Drijfhout for the peptide synthesis, Ludvig Sollid for generating and kindly providing us with the pET14b_29-35 $\gamma 5$ gliadin plamid, Peter van Veelen and George Janssen for the mass spectrometry of the recombinant $\gamma$-gliadin, Iris Janssen for the animal care, and Nadine van Montfoort for providing chloroquine. This research was funded by the Dutch Sophia Research Foundation grants: 557 and 608, an Erasmus MC Mrace pilot grant, and the Dutch Celiac Disease Consortium (CDC2).

\section{DISCLOSURE}

The authors declared no conflict of interest.

c 2017 Society for Mucosal Immunology

\section{REFERENCES}

1. Green, P.H. \& Cellier, C. Celiac disease. N. Engl. J. Med. 357, 1731-1743 (2007).

2. Shan, L. et al. Identification and analysis of multivalent proteolytically resistant peptides from gluten: implications for celiac sprue. J. Proteome Res. 4, 1732-1741 (2005).

3. Shan, L. et al. Structural basis for gluten intolerance in celiac sprue. Science 297, 2275-2279 (2002).

4. Molberg, 0 . et al. Tissue transglutaminase selectively modifies gliadin peptides that are recognized by gut-derived $\mathrm{T}$ cells in celiac disease. Nat. Med. 4, 713-717 (1998).

5. van de Wal, Y. et al. Selective deamidation by tissue transglutaminase strongly enhances gliadin-specific T cell reactivity. J. Immunol. 161, 15851588 (1998).

6. van Leeuwen, M.A. et al. Increased production of interleukin-21, but not interleukin-17A, in the small intestine characterizes pediatric celiac disease. Mucosal Immunol. 6, 1202-1213 (2013).

7. Fina, D. et al. Interleukin 21 contributes to the mucosal T helper cell type 1 response in coeliac disease. Gut 57, 887-892 (2008).

8. Nilsen, E.M., Lundin, K.E., Krajci, P., Scott, H., Sollid, L.M. \& Brandtzaeg, P. Gluten specific, HLA-DQ restricted T cells from coeliac mucosa produce cytokines with Th1 or Th0 profile dominated by interferon gamma. Gut 37, 766-776 (1995).

9. Bodd, M. et al. HLA-DQ2-restricted gluten-reactive T cells produce IL-21 but not IL-17 or IL-22. Mucosal Immunol. 3, 594-601 (2010).

10. Du Pre, M.F. et al. Tolerance to ingested deamidated gliadin in mice is maintained by splenic, type 1 regulatory T cells. Gastroenterology 141, 610-620, 620.e611-612 (2011).

11. Broere, F. et al. Cyclooxygenase-2 in mucosal DC mediates induction of regulatory $\mathrm{T}$ cells in the intestine through suppression of IL-4. Mucosal Immunol. 2, 254-264 (2009).

12. Coombes, J.L. et al. A functionally specialized population of mucosal CD103 + DCs induces Foxp3 + regulatory T cells via a TGF-beta and retinoic acid-dependent mechanism. J. Exp. Med. 204, 1757-1764 (2007).

13. Hauet-Broere, F., Unger, W.W., Garssen, J., Hoijer, M.A., Kraal, G. \& Samsom, J.N. Functional CD25- and CD25 + mucosal regulatory T cells are induced in gut-draining lymphoid tissue within $48 \mathrm{~h}$ after oral antigen application. Eur. J. Immunol. 33, 2801-2810 (2003).

14. Sun, C.M. et al. Small intestine lamina propria dendritic cells promote de novo generation of Foxp3 T reg cells via retinoic acid. J. Exp. Med. 204, 1775-1785 (2007). 
15. Delamarre, L., Pack, M., Chang, H., Mellman, I. \& Trombetta, E.S. Differential lysosomal proteolysis in antigen-presenting cells determines antigen fate. Science 307, 1630-1634 (2005).

16. Jung, J.Y., Roberts, L.L. \& Robinson, C.M. The presence of interleukin-27 during monocyte-derived dendritic cell differentiation promotes improved antigen processing and stimulation of T cells. Immunology 144, 649-660 (2015).

17. Pot, C. et al. Cutting edge: IL-27 induces the transcription factor C-Maf, cytokine IL-21, and the costimulatory receptor ICOS that coordinately act together to promote differentiation of IL-10-producing Tr1 cells. J. Immunol. 183, 797-801 (2009).

18. Kraal, G. \& Mebius, R. New insights into the cell biology of the marginal zone of the spleen. Int. Rev. Cytol. 250, 175-215 (2006).

19. Van Rooijen, N. \& Sanders, A. Liposome mediated depletion of macrophages: mechanism of action, preparation of liposomes and applications. J. Immunol. Methods 174, 83-93 (1994).

20. van Rooijen, N., Sanders, A. \& van den Berg, T.K. Apoptosis of macrophages induced by liposome-mediated intracellular delivery of clodronate and propamidine. J. Immunol. Methods 193, 93-99 (1996).

21. Awasthi, A. et al. A dominant function for interleukin 27 in generating interleukin 10-producing anti-inflammatory Tcells. Nat. Immunol. 8, 13801389 (2007).

22. Berton, G., Mocsai, A. \& Lowell, C.A. Src and Syk kinases: key regulators of phagocytic cell activation. Trends Immunol. 26, 208-214 (2005).

23. Mizuochi, T., Yee, S.T., Kasai, M., Kakiuchi, T., Muno, D. \& Kominami, E. Both cathepsin B and cathepsin D are necessary for processing of ovalbumin as well as for degradation of class II MHC invariant chain. Immunol. Lett. 43, 189-193 (1994).
24. Sollid, L.M., Qiao, S.W., Anderson, R.P., Gianfrani, C. \& Koning, F. Nomenclature and listing of celiac disease relevant gluten T-cell epitopes restricted by HLA-DQ molecules. Immunogenetics 64, 455460 (2012).

25. Bethune, M.T. \& Khosla, C. Parallels between pathogens and gluten peptides in celiac sprue. PLoS Pathog. 4, e34 (2008).

26. Jonuleit, H., Schmitt, E., Schuler, G., Knop, J. \& Enk, A.H. Induction of interleukin 10-producing, nonproliferating $\mathrm{CD} 4(+)$ T cells with regulatory properties by repetitive stimulation with allogeneic immature human dendritic cells. J. Exp. Med. 192, 1213-1222 (2000).

27. Roncarolo, M.G., Gregori, S., Battaglia, M., Bacchetta, R., Fleischhauer, K. \& Levings, M.K. Interleukin-10-secreting type 1 regulatory Tcells in rodents and humans. Immunol. Rev. 212, 28-50 (2006).

28. Ansari, N.A. etal. IL-27 and IL-21 are associated with Tcell IL-10 responses in human visceral leishmaniasis. J. Immunol. 186, 3977-3985 (2011).

29. Forsberg, G., Hernell, O., Melgar, S., Israelsson, A., Hammarstrom, S. \& Hammarstrom, M.L. Paradoxical coexpression of proinflammatory and down-regulatory cytokines in intestinal T cells in childhood celiac disease. Gastroenterology 123, 667-678 (2002).

30. Gianfrani, C. et al. Gliadin-specific type 1 regulatory T cells from the intestinal mucosa of treated celiac patients inhibit pathogenic $T$ cells. J. Immunol. 177, 4178-4186 (2006).

31. Borrelli, M. et al. Immunoregulatory pathways are active in the small intestinal mucosa of patients with potential celiac disease. Am. J. Gastroenterol. 108, 1775-1784 (2013).

32. Hmida, N.B. et al. Impaired control of effector T cells by regulatory T cells: a clue to loss of oral tolerance and autoimmunity in celiac disease? Am. J. Gastroenterol. 107, 604-611 (2012). 